\title{
A coupled BEM/FEM formulation for drop interaction in Stokes flows with flexible and slip confining boundaries
}

\author{
Delfim Soares $\mathrm{Jr}^{1 *}$ and Luiz C. Wrobel ${ }^{2}$ \\ ${ }^{1}$ Structural Engineering Department, Federal University of Juiz de Fora, Juiz de Fora, MG, Brazil \\ ${ }^{2}$ Institute of Materials and Manufacturing, Brunel University London, Uxbridge UB8 3PH, UK
}

\begin{abstract}
In this work, fluid-fluid-solid coupled models are analysed, considering the interaction of boundary and finite element techniques. In this context, the paper focuses on the study of deforming drops through bulk fluids bounded by flexible walls. Here, the fluid subdomains are assumed to be viscous and incompressible, and they are modelled by the BEM. The solid subdomains are assumed to be elastic, and they are modelled by the FEM. Both discontinuity of tractions on the fluid-fluid common boundaries and discontinuity of velocities on the fluidsolid interfaces are considered. For the discontinuity of velocities, a formulation based on nonlinear slip boundary conditions is adopted, which is treated employing a relaxed iterative approach. A Lagrangian representation is considered and remeshing is applied on the fluidfluid interfaces, reducing the appearance of numerical problems. Numerical results are presented to illustrate the performance and potentialities of the proposed techniques.
\end{abstract}

Keywords: Coupled analysis; Boundary element method; Finite element method; Nonlinear slip boundary conditions; Remeshing; Drop deformation.

\footnotetext{
* Corresponding author. E-mail: delfim.soares@ufjf.edu.br; Tel: +55 3221023468.
} 


\section{Introduction}

The main focus of the work described in this paper is to develop a fluid-fluid-solid coupled model to study the interaction between deforming drops transported by bulk fluids bounded by rigid or flexible walls. Practical engineering applications of this work involve, for instance, the biomedical problem of transport of drops or bubbles in arteries. One interesting such application is the use of gas embolotherapy to treat tumours [34, 35]. In this potential kind of treatment, which is still under development, transvascular perfluorocarbon droplets, around 6 $\mu \mathrm{m}$ in diameter, are selectively vaporised using high intensity ultrasound at a desired location near or in the tumor microcirculation to form substantially larger gas bubbles (around 150 times volume expansion), which then occlude blood flow and induce tumour infarction. The homogeneity of bubble delivery is expected to determine the uniformity of tumour infarction. Consequently, understanding the transport of bubbles, which are long in this case compared to microvessel diameters, is essential to designing treatment strategies. Because of the geometrical scales involved, the bulk fluid may be able to slip at the solid boundaries. Calderon et al. [36] and Eshpuniyani et al. [37] developed BEM models for bubble propagation in microvessels considering slip at the walls, but assuming the walls to be rigid.

Another practical engineering application of the current technique is for fluid flow in micro and nano-fluidic rotating devices. Nieto et al. [38] developed a BEM formulation for Stokes flow with linear slip to study the flow in micro-devices such as concentric and eccentric rotating Couette mixers, and a single rotor mixer, again considering rigid walls.

Several fluid-solid interaction models have been previously developed, some of which involve coupled models based on the finite element method (FEM) to model the solid and the boundary element method (BEM) to model the fluid. Although these approaches can be useful in addressing many engineering problems, they mostly correspond to standard direct coupling methodologies and thus exhibit well-known limitations. Indeed, the direct coupling of two distinct methods generally involves assembling a single system matrix, accounting for the contributions of each method and for the required coupling interface conditions, which frequently becomes poorly conditioned due to the different nature of the methods. Since this system is formed from the contributions of distinct methods, it is also usually not possible to make use of their individual advantages in terms of optimised solvers or memory storage (e.g. the final system of equations in coupled BEM-FEM models will no longer be banded and symmetric, etc.). In addition to this limitation, by forming a single system of equations, a very 
large problem usually arises, leading to increased computational efforts and thus to a loss of performance.

The above limitations have justified the appearance of iterative algorithms to obtain accurate solutions in a more efficient manner. Perhaps one of the first iterative techniques for general problems is the Schwarz alternating strategy [1, 2], in which the domain of analysis is partitioned into overlapping subdomains and the solution is found by successively iterating along these subdomains until convergence is reached. This classical and simple to implement algorithm has been applied to many problems, including potential problems [3] and electromagnetic wave propagation problems [4].

In recent years, several iterative domain decomposition techniques have been proposed for a wide range of problems, providing more flexibility and efficiency. These techniques usually consider the analysis of coupled models, taking into account the interaction of different physical phenomena and discretisation methods. In fact, for complex models, iterative domain decomposition techniques are recommended. Indeed, a proper numerical simulation is hardly achieved by a single numerical technique in those cases, mostly because complex and quite different phenomena interact, requiring particular advanced expertise, and/or large scale problems are involved, demanding high computational efforts. Several algorithms are available nowadays discussing iterative non-overlapping partitioned analysis. Rice et al. [5] presented a comprehensive discussion on several interface relaxation procedures for elliptic problems, comparing formulations and performances.

One of the first publications on BEM-FEM iterative coupling was presented by Lin et al. [6], who discussed a relaxed iterative procedure considering linear static stress analyses. Similar approaches have also been presented for potential and mechanical static linear analyses [7, 8]. In the works of Elleithy et al. [9, 10], addressing potential and elastostatic problems, the authors propose that the domain of the original problem is subdivided into subdomains, and the coupling between the different subdomains is performed by using smoothing operators on the interface boundaries. Their strategy allows separate computations for the BEM and FEM subdomains, with successive updating of the boundary conditions at the interfaces until convergence is achieved. Similar approaches for the analysis of different linear problems using domain decomposition techniques were also presented in [11, 12]. Further developments of these strategies to nonlinear analysis in solid mechanics can be found in Elleithy et al. [13], using an interface relaxation BEM-FEM coupling method for elastostatic analysis; Jahromi et al. [14], who established a coupling procedure based on a 
sequential iterative Dirichlet-Neumann coupling algorithm for nonlinear soil-structure interaction; or Soares and Godinho [15], who considered coupled inelastic models with adaptive remeshing. Taking into account iterative formulations, interacting fluid-solid models have also been analysed considering both BEM-FEM [16] and BEM-BEM [17] coupled techniques; however, these formulations are restricted to simulate the behaviour of acoustic fluids (reviews on the topic are provided in $[18,19]$ ).

The numerical model developed in this work also considers the interaction of BEM and FEM techniques. The fluid subdomains (bulk fluid and drops) are modelled by the BEM, and assumed to be viscous and incompressible. The solid subdomain is assumed to be elastic, and is modelled by the FEM. Both discontinuity of tractions on the fluid-fluid common boundaries and discontinuity of velocities on the fluid-solid interfaces are considered. For the discontinuity of velocities, a formulation based on nonlinear slip boundary conditions is adopted [20], which is treated in the present work by employing a relaxed iterative approach. A Lagrangian representation is considered and remeshing is applied on the fluid-fluid interfaces, to prevent the appearance of numerical problems.

The justification to introduce slip between the solid and fluid boundaries is due to the geometrical scale of the biomedical problem of interest to the authors. It has been demonstrated that, at the micro/nano scales, the mechanical properties at the fluid-solid interface cannot be understood by simply extrapolating known properties of the bulk fluid [21]. Experimental [22, 23], theoretical [24] and numerical [25, 26] simulations at micro/nano scales have provided clear evidence that wall slip occurs at fluid-solid interfaces, and show that the degree of boundary slip is a function of the liquid viscosity and the shear rate. Variation in slip length arises from the fact that, during a collision with a solid surface, a fluid molecule will transfer some of its tangential momentum to the solid. The collision frequency is not high enough to ensure thermodynamic equilibrium, and a certain degree of slip tangential velocity must be allowed [27].

A Lagrangian representation is considered for this moving boundary problem and remeshing is applied on the fluid-fluid interfaces, following the algorithms proposed in [28]. The present work focuses on 2D models and numerical results are presented to illustrate the performance and potentialities of the proposed techniques. To the best of the authors' knowledge, a BEM formulation considering slip and flexible walls has not been presented before. 


\section{Governing equations and numerical modelling}

In this section, the basic governing equations of the fluid-fluid-solid interacting model are discussed, as well as their discretizations by boundary and finite element techniques. Some special procedures employed to improve the performance of the proposed numerical approach, such as interface remeshing, relaxed iterative procedures, etc, are also described here.

\subsection{Fluid subdomain}

In this work, fluids are assumed to be viscous and incompressible, and to flow at very low velocities. Thus, low Reynolds number flows are studied and inertia terms in the NavierStokes equations can be neglected, so that the system is in a state of creeping motion. In this case, the conservation of mass and momentum is given by the equations:

$$
\begin{gathered}
u_{i, i}(\mathbf{x}, t)=0 \\
\sigma_{i j, j}(\mathbf{x}, t)=0
\end{gathered}
$$

where $t$ stands for time (as previously highlighted, the acceleration term is neglected in equation (2), so a quasi-steady formulation is adopted), $\mathbf{x}$ stands for the position vector, $\mathbf{u}$ represents the velocity vector and $\boldsymbol{\sigma}$ is the stress tensor, with indicial notation adopted throughout the paper. The fluids are assumed to be Newtonian, so that:

$$
\sigma_{i j}(\mathbf{x}, t)=-p(\mathbf{x}, t) \delta_{i j}+\mu \varepsilon_{i j}(\mathbf{x}, t)
$$

where $p$ is the hydrostatic pressure, $\mu$ is the viscosity of the fluid, $\boldsymbol{\varepsilon}$ stands for the strain rate tensor, and $\boldsymbol{\delta}$ stands for the Kronecker delta tensor.

The system of equations (1)-(3) must be supplemented by appropriate boundary conditions. The simplest boundary conditions that may be considered are those that directly prescribe velocities or tractions ( $\tau_{i}=\sigma_{i j} n_{j}$, where $\mathbf{n}$ stands for a unit direction vector) on the boundaries of the fluid domain. However, more elaborate conditions may become necessary if more complex physical phenomena are to be represented. This is the case of the current fluidfluid and fluid-solid interactions, and these more advanced boundary conditions are discussed 
in detail in the sections that follow. In the next subsection, the Boundary Element Method (BEM) is briefly presented.

\subsubsection{BEM formulation}

Here, a standard BEM approach is considered. The boundary integral equation that describes the velocity field at a given source point $\chi$ can be written as follows:

$$
c_{i j}(\chi) u_{j}(\chi, t)=\frac{1}{\mu} \int_{\Gamma} u_{i j}^{*}(\chi, \mathbf{x}) \tau_{j}(\mathbf{x}, t) d \Gamma-\int_{\Gamma} \tau_{i j}^{*}(\chi, \mathbf{x}) u_{j}(\mathbf{x}, t) d \Gamma
$$

where $\mathbf{u}^{*}$ and $\boldsymbol{\tau}^{*}$ stand for the steady-state fundamental tensors (velocity and traction, respectively), c stands for the free term tensor, and $\Gamma$ represents the boundary of the model.

Once discretization is introduced and proper numerical treatment is considered, the following system of equations arises, describing the BEM solution of the fluid subdomain:

$$
\mathbf{H u}=\mathbf{G} \boldsymbol{\tau}
$$

where $\mathbf{G}$ and $\mathbf{H}$ stand for influence matrices. By re-arranging the system of equations (5), taking into account the boundary conditions of the problem, just known and unknown variables can be moved to the right and left hand sides of the system of equations, respectively, allowing its solution. Equations (4)-(5) only intend to summarily describe the boundary element formulation considered here; for further details on the topic, the following references are suggested $[29,30]$.

\subsection{Fluid-fluid interaction}

Drops of viscous fluids in a carrying bulk fluid are considered here. In this case, the fluidfluid interactions can be described by the interface conditions acting on the drops surfaces; i.e., the interactions are governed by the compatibility and equilibrium conditions along the fluid interfaces.

The compatibility and equilibrium conditions at the interface between a drop and the bulk fluid are given by:

$$
u_{i}^{f}(\mathbf{x}, t)-u_{i}^{d}(\mathbf{x}, t)=0
$$




$$
\tau_{i}^{f}(\mathbf{x}, t)-\tau_{i}^{d}(\mathbf{x}, t)=\gamma \kappa(\mathbf{x}, t) n_{i}(\mathbf{x}, t)
$$

where the superscripts $d$ and $f$ indicate if a variable is related to the drop or to the bulk fluid subdomain, respectively, $\gamma$ stands for the surface tension coefficient, $\kappa$ is the surface curvature, and $\mathbf{n}$ is the unit drop-outward normal vector. As one can observe, equation (6a) represents the continuity of the velocities, whereas equation (6b) represents the continuity of the tangential stresses and the discontinuity of the normal stresses, which is caused by an interfacial tension. In the next subsection, equations (6) are employed to define the adopted BEM-BEM coupling approach. This coupled procedure is based on a BEM subregion formulation.

\subsubsection{BEM-BEM coupling}

It is possible to combine two integral equations (see expression (4)), one for source points belonging to the bulk fluid and the other for source points belonging to a drop, in order to eliminate the interface tractions between these subdomains by using the equilibrium equation (6b). By doing so, the following integral equation arises:

$$
\begin{aligned}
\eta(\chi) c_{i j}(\chi) u_{j}(\chi, t) & =\frac{1}{\mu^{f}} \int_{\Gamma^{e}} u_{i j}^{*}(\chi, \mathbf{x}) \tau_{j}(\mathbf{x}, t) d \Gamma-\int_{\Gamma^{e}} \tau_{i j}^{*}(\chi, \mathbf{x}) u_{j}(\mathbf{x}, t) d \Gamma+ \\
& +\frac{\gamma}{\mu^{f}} \int_{\Gamma^{d}} u_{i j}^{*}(\chi, \mathbf{x}) \kappa(\mathbf{x}, t) n_{j}(\mathbf{x}, t) d \Gamma-\left(1-\frac{\mu^{d}}{\mu^{f}}\right) \int_{\Gamma^{d}} \tau_{i j}^{*}(\chi, \mathbf{x}) u_{j}(\mathbf{x}, t) d \Gamma
\end{aligned}
$$

where $\Gamma^{d}$ and $\Gamma^{e}$ represent the internal (drop) and the external boundary of the bulk fluid, respectively (i.e., $\left.\Gamma^{f}=\Gamma^{d} \cup \Gamma^{e}\right)$, and $\eta(\chi)=1$ for $\chi \in \Gamma^{e}$ and $\eta(\chi)=1+\left(\mu^{d} / \mu^{f}\right)$ for $\chi \in \Gamma^{d}$.

Once equation (7) is established, discretization by boundary elements can be considered, leading to an algebraic system of equations analogous to equation (5), whose solution describes the behaviour of the variables of the fluid subdomains. Thus, the velocities along the drops surfaces can be computed, as well as the unknown fields on $\Gamma^{e}$.

In a Lagrangian representation, the fluid-fluid interface is assumed to deform with the interface velocity, such that the location of the boundaries describing the drops must be continuously adapted, following the relation $\dot{\mathbf{x}}=\mathbf{u}$. Thus, in addition to the solution of the BEM system of equations, a time stepping procedure must be considered in order to compute the evolution of the fluid-fluid interfaces. This can be carried out as follows: 


$$
\mathbf{x}^{t+\Delta t}=\mathbf{x}^{t}+\mathbf{u}^{t} \Delta t, \text { for } \mathbf{x} \in \Gamma^{d}
$$

where, in this case, a simple finite difference expression is employed and $\Delta t$ stands for the adopted time-step.

Since the evolution of the positions of the nodes related to the drops is computed as described in equation (8), an effective node relocation routine along the fluid interfaces becomes of great importance in order to avoid numerical problems such as singularities due to an excessive approximation of boundary nodes, etc. In the next subsection, a numerical procedure to relocate the nodes on the drops surfaces is discussed.

\subsubsection{Remeshing procedures}

As it is well known, taking into account a natural coordinate $\xi$, the physical coordinates of a line (2D models are focused here, thus the drop surfaces are described by lines) can be represented as follows:

$$
x_{i}=\sum_{n=1}^{m} N(\xi)^{n} x_{i}
$$

where ${ }^{n} N$ stands for an interpolation function and ${ }^{n} \mathbf{x}$ represents the position of the $n^{\text {th }}$ node (in a total of $m$ nodes) describing the line in focus.

The main goal here is to obtain the middle point location of this line, so that this information can guide the node relocation. In order to do so, the following auxiliary values can be defined, taking into account the extremities of the line, in each direction: (i) $\hat{x}_{i}=\frac{1}{2}\left({ }^{m} x_{i}+{ }^{1} x_{i}\right)$; and (ii) $\Delta x_{i}=\left({ }^{m} x_{i}-{ }^{1} x_{i}\right)$. Then, one can find $\hat{\xi}_{i}$ that solves equation (9) for each $\hat{x}_{i}$ and, once these values are computed, the natural coordinate related to the middle point of the line can be easily approximated as an average of these values:

$$
\bar{\xi}=\sum_{i} \Delta x_{i} \hat{\xi}_{i} / \sum_{i} \Delta x_{i}
$$

Once $\bar{\xi}$ is computed, the physical coordinates of the middle point of the line (i.e., $\bar{X}_{i}$ ) can be easily established following equation (9). 
In this work, a group of $m$ consecutive nodes (where $m$ is an odd integer) is considered to define a local line over the surface of the drops, and the entire surface is divided into local lines, each with $m$ nodes. Then, the middle nodes defining these local lines are relocated to their middle positions, following the computed $\bar{x}_{i}$ values for each line. In the sequence, the definitions of the local lines are translated by one node (i.e., the first nodes of the local lines become the consecutive nodes of the previously defined first nodes) and the procedure is repeated, once again relocating the middle nodes of the local lines. After a total of $m$ adjacent translations of the local lines, the adopted remeshing procedure is completed. In this work, $m$ $=3$ is adopted; thus, second-order polynomials may describe the interpolation functions $N$ and $\hat{\xi}_{i}$ can be simply computed using the Bhaskara formula [28].

In Fig. 1, the effectiveness of the proposed remeshing procedure is illustrated, taking into account a deformed drop discretized by the BEM. In the analysis related to Fig. 1(a), the remeshing procedure is active and a regular distribution of the BEM nodes is observed. On the other hand, in the analysis carried out in Fig. 1(b), the remeshing procedure is not considered, rendering an excessive concentration of nodes at the drop extremities, allowing numerical difficulties to arise.

\subsection{Solid subdomain}

For the solid subdomains, the conservation of momentum is given by:

$$
\sigma_{i j, j}(\mathbf{x}, t)=0
$$

where null body forces are considered and, again, the acceleration term is neglected, so that a quasi-static formulation is adopted. Here, elastic constitutive relations are employed:

$$
\sigma_{i j}(\mathbf{x}, t)=\frac{E}{(1+v)}\left[\frac{v}{(1-2 v)} \delta_{i j} \varepsilon_{k k}(\mathbf{x}, t)+\varepsilon_{i j}(\mathbf{x}, t)\right]
$$

where $E$ and $v$ represent the Young's modulus and the Poisson's ratio, respectively, and $\boldsymbol{\varepsilon}$ stands for the strain tensor.

As in section 2.1, the system of equations (11)-(12) must be supplemented by appropriate boundary conditions, and prescribed displacements or tractions are considered here. The interconnection of these boundary conditions with the fluid subdomain is discussed in section 2.4. In the next subsection, the Finite Element Method (FEM) is briefly presented. 


\subsubsection{FEM formulation}

Here, a standard FEM approach is considered. The integral weak-form of the governing equations (11) can be written as:

$$
\int_{\Omega} \sigma_{i j}(\mathbf{x}, t) w_{i k, j}(\mathbf{x}) d \Omega-\int_{\Gamma_{\tau}} \tau_{i}(\mathbf{x}, t) w_{i k}(\mathbf{x}) d \Gamma=0
$$

where $\Omega$ and $\Gamma$ represent the domain and the boundary of the solid, respectively, and $\Gamma=\Gamma_{v} \cup \Gamma_{\tau}$, where $\Gamma_{v}$ stands for the Dirichlet boundary and $\Gamma_{\tau}$ stands for the Neumann boundary. The variable $\boldsymbol{w}$ stands for a weight tensor, which is assumed to have null values on the Dirichlet boundary.

By introducing spatial approximations for the variables of the model into the integral equation (13), and by adopting these approximations to define the specified weight functions (Galerkin Method), the following system of equations can be obtained, once relations (12) are employed (as well as the strain tensor definition as function of the displacements) and proper numerical treatment is considered:

$$
\mathbf{K v}=\mathbf{f}
$$

where $\mathbf{K}$ stands for the stiffness matrix, and $\mathbf{v}$ and $\mathbf{f}$ stand for the nodal displacement and force vector, respectively. After considering the Dirichlet boundary conditions of the problem, the FEM responses for the elastic solid can be computed, following equation (14). Equations (13)-(14) only intend to summarily describe the finite element formulation considered here; for further details on the topic, the following references are suggested [31, 32].

\subsection{Fluid-solid interaction}

Analogously to the fluid-fluid interaction, the fluid-solid interaction is also governed by the compatibility and equilibrium conditions along the common interfaces. These conditions are given by:

$$
\begin{aligned}
& x_{i}^{f}(t)-x_{i}^{s}(t)=0 \\
& \tau_{i}^{f}(\mathbf{x}, t)+\tau_{i}^{s}(\mathbf{x}, t)=0
\end{aligned}
$$


where the superscripts $s$ and $f$ indicate if a variable is related to the solid or to the bulk fluid subdomain, respectively. As one can observe, equation (15a) represents the continuity of the model, indicating that the fluid keeps in touch with the solid, and equation (15b) represents the equilibrium of the interface boundary forces. In the next subsection, these equations are employed to define the adopted BEM-FEM coupling approach. A pseudo-coupled formulation is adopted here, and the BEM and FEM subdomains are treated and solved separately, as uncoupled models. In addition to equations (15), relations between the velocities of the fluid and solid subdomains along their common boundaries must also be defined. In subsection 2.4.2, slip boundary conditions are discussed in detail, allowing the discontinuity of velocities at the fluid-solid interfaces.

\subsubsection{BEM-FEM coupling}

The fluid-solid interaction can be uncoupled through the time stepping procedure, allowing each subdomain to be analysed separately. Thus, the fluid subdomains can be analysed considering prescribed velocities on the fluid-solid interfaces (further details about these velocities are given in the next subsection) and the tractions on these interfaces can be computed following the discussions presented in sections 2.1 and 2.2. Once these tractions are computed, they can be applied as prescribed boundary conditions for the solid subdomains, following the equilibrium relation (15b). Thus, equation (13) can be rewritten as:

$$
\int_{\Omega} \sigma_{i j}(\mathbf{x}, t) w_{i k, j}(\mathbf{x}) d \Omega-\int_{\Gamma_{\tau}^{e}} \tau_{i}(\mathbf{x}, t) w_{i k}(\mathbf{x}) d \Gamma+\int_{\Gamma_{\tau}^{i}} \tau_{i}^{f}(\mathbf{x}, t) w_{i k}(\mathbf{x}) d \Gamma=0
$$

which explicitly shows the influence of the bulk fluid subdomain over the solid subdomain. In this case, $\Gamma_{\tau}^{i}$ and $\Gamma_{\tau}^{e}$ represent the internal (interface) and the external Neumann boundary of the solid, respectively (i.e., $\Gamma_{\tau}=\Gamma_{\tau}^{i} \cup \Gamma_{\tau}^{e}$ ).

Once equation (16) is established, the FEM system of equations can be computed (analogously to equation (14)) and the solid subdomains can be solved, allowing computing their deformations. In the sequence, the geometry of the solid subdomains can be updated taking into account the computed displacements, while the geometry of the bulk fluid interfaces is also updated following the continuity relation (15a):

$$
\mathbf{x}^{t+\Delta t}=\mathbf{x}^{t}+\mathbf{v}^{t}, \text { for } \mathbf{x} \in \Gamma^{s}
$$


where $\Gamma^{s}$ stand for the bulk fluid boundaries that interface the solid subdomains $\left(\Gamma^{s} \subset \Gamma^{e}\right.$, for the bulk fluid). Since small deformations are supposed to occur in the solid subdomains, remeshing procedures are not necessary.

\subsubsection{Slip boundary condition}

The Navier slip boundary condition states that the relative tangential fluid velocity, with respect to the tangential solid velocity, is proportional to the tangential projection of the local shear rate $(e)$ :

$$
\begin{aligned}
& u_{i}(\mathbf{x}, t)-\dot{v}_{i}(\mathbf{x}, t)=L_{s}(\mathbf{x}, t) e(\mathbf{x}, t) s_{i}(\mathbf{x}, t) \\
& e(\mathbf{x}, t)=s_{i}(\mathbf{x}, t)\left(u_{i, j}(\mathbf{x}, t)+u_{j, i}(\mathbf{x}, t)\right) n_{j}(\mathbf{x}, t)
\end{aligned}
$$

where $\mathbf{n}$ and s stand for normal and tangential unit vectors, respectively, and $L_{s}$ represents the slip length, which may also depend on the tangential shear rate at the solid surface, as indicated below:

$$
L_{s}(\mathbf{x}, t)=b(1-\beta e(\mathbf{x}, t))^{-a / 2}
$$

In equation (19), $a$ is an index that depends on the cohesive property at the interface, $b$ is the slip length in the case of linear slip condition, and $\beta$ is function of the critical shear rate. The nonlinear slip boundary condition is obtained by substituting equation (19) into equation (18a). The linear slip condition and the no-slip condition can be regarded as particular cases of equation (19). Thus, the linear slip condition can be obtained by adopting $\beta=0$ (in this case, $L_{s}=b$ ) and the no-slip condition can be obtained by adopting $b=0$.

Once equations (18) are established, one can observe that the prescribed velocities for the bulk fluid, on the fluid-solid interfaces, become function of the fluid tractions on those interfaces, as there is a direct correlation between the tangential projection of the local shear rate and the tangential traction, as well as of the solid velocities themselves, which can be easily evaluated taking into account simple finite difference expressions based on the evolution of the solid vector positions (analogously to expression (8), for instance).

Since equations (18)-(19) represent a nonlinear configuration, an iterative procedure must be introduced into the analysis, in order to properly handle their evaluations. In order to do so, the present work adopts the following iterative algorithm: 


$$
\begin{aligned}
& u_{i}^{(k+1)}-\dot{v}_{i}=L_{s}^{(k)} e^{(k+1)} S_{i} \\
& L_{s}^{(k+1)}=b\left(1-\beta e^{{ }^{(k+1)}}\right)^{-\alpha / 2} \\
& e^{\prime(k+1)}=(\alpha) e^{(k+1)}+(1-\alpha) e^{\prime^{(k)}}
\end{aligned}
$$

where the superscript $(k)$ stands for the iterative step of the analysis.

As described by equation (20a), the $L_{s}$ variable in equation (18a) is computed based on the information from the previous iterative step, and a relaxed value of $e$ is considered in this case, i.e., the value of $e^{\prime}$ is considered instead of $e$ itself, as indicated in equation (20b). The definition of $e^{\prime}$ is provided in equation (20c), which employs a relaxation parameter $\alpha$. Here, $\alpha \neq 1$ is adopted in order to speed up and/or to ensure the convergence of the iterative algorithm. Of course, this iterative algorithm must only be activated once $\beta \neq 0$ is considered, otherwise linear boundary conditions are enabled and no iterative procedures are necessary.

A workflow diagram is presented in Fig.2, briefly summarising the sequence of the proposed calculations discussed in this section.

\section{Numerical applications}

In this section, two numerical applications are considered to illustrate the performance of the proposed numerical methodologies. In the first application, the flow through a channel is analysed. Initially, a simple horizontal geometry is adopted for the channel and, in the sequence, a forward-facing step is introduced into the configuration of the model. In both cases, the analyses of the interaction of the bulk fluid with flowing drops, considering rigid and flexible walls for the channel, as well as nonlinear, linear and no-slip boundary conditions, are provided. In the second application, analogous analyses are carried out for a lid-driven cavity flow. In this work, analyses accounting for the possibility of merging two or more drops are not considered.

In the applications that follow, quadratic boundary elements and linear finite elements are considered. For the iterative procedure related to the nonlinear slip boundary conditions, convergence is achieved once the relative error of the tractions on the fluid-solid interfaces is lower than a given value, and a tight tolerance of $10^{-5}$ is adopted here. For the value of the relaxation parameter, usually good results are obtained with $\alpha=1$; however, when this is not the case, $\alpha=1-\beta$ can be selected, increasing the relaxing feature of the technique as the 
nonlinearity of the model increases. In addition, in the sections that follow, the international system of units is adopted and an explicit indication of units is omitted.

\subsection{Flow through a channel}

As previously remarked, initially, a simple horizontal channel is considered. A sketch of the model is provided in Fig. 3(a). The geometry of the channel is defined by a length of 1, a height of 0.2 and a thickness of 0.01 (horizontal walls). In this application, unity values of the horizontal tractions are applied on the vertical boundaries of the bulk fluid, i.e., at $x_{1}=0$ and at $x_{1}=1$. The physical properties of the model are: $\mu^{f}=10^{-3} ; \mu^{d}=2 \cdot 10^{-3} ; \gamma=10^{-2} ; E=10^{5} ; v=$ 0.25. The external contour of the bulk fluid is discretized by $240 \mathrm{BE}$ (200 BE on the fluidsolid interface) and each drop surface is discretized by $30 \mathrm{BE}$; the walls of the channel are discretized by 800 square FE. Each drop has a diameter of 0.05 and their centres are located at $(0.15 ; 0.00)$ and $(0.20 ; 0.05)$, see Fig. 3. A time-step of $10^{-3}$ is adopted for the analyses.

For this simple configuration and considering the absence of drops on the carrying fluid, as well as considering rigid solid walls, analytical solutions are available for the velocity profile of the bulk fluid, taking into account several slip boundary conditions [33]. In Fig. 4, these profiles are depicted, taking into account analytical solutions and computed results, illustrating the good accuracy of the adopted numerical techniques. The parameters describing the slip boundary conditions are: (i) no-slip ( $b=0 ; \beta=0)$; (ii) linear ( $b=0.1 ; \beta=0$ ); and (iii) nonlinear ( $b=0.1 ; \beta=0.4$ ). In this work, $a=1$ is always considered. As one can observe in Fig. 4, slip boundary conditions, as expected, increase the values of the fluid velocities in the channel, and a greater increase is observed when the nonlinear model is considered.

As a sequence to the analyses of channel flows, more complex configurations may now be considered. Thus, firstly, one drop, which is initially centred at $(0.15 ; 0.00)$, is inputted into the analysis and its behaviour within the horizontal channel is studied. The evolution of the drop deformation, considering different slip boundary conditions, is depicted in Figs. 5 and 6, taking into account rigid and flexible walls for the channel, respectively. In these figures, different shades of gray are adopted to represent the time evolution of the results; thus, darker colours are related to more recent time instants. In Fig. 7, the evolution of the horizontal position of the mass centre of the drop is depicted, once again considering rigid (BEM analysis) and flexible (BEM-FEM analysis) walls.

As one can observe in Fig. 7, taking into account the tangent values of the described curves, the drop basically moves with the same velocity at the centre of the bulk fluid. 
Considering rigid walls, this velocity is practically constant whereas, considering flexible walls, it decreases as the wall expands and it increases as the wall contracts. In addition, the velocity of the drop considerably increases as slip boundary conditions are considered. These phenomena are expected, and the adopted numerical techniques properly reproduce them.

Similar analyses are now carried out considering two drops within the horizontal channel, as shown in Figs. 8 and 9. The results presented in Fig. 8 consider a channel with rigid walls whereas, in Fig. 9, the computed evolution of the drops is illustrated considering flexible walls. It can be seen that the second drop affects the trajectory of the central drop, and its previously straight movement in a horizontal line is modified, including a subtle movement in the vertical direction as well. Figs. 8 and 9 also indirectly illustrate the parabolic profile of the velocities along the carrying fluid, with the drops deforming following this outline. In this context, it is interesting to observe that the new second drop is more intensively deformed when it flows through the contracted stretch of the channel with flexible walls (as well as it is less intensively deformed when it flows through the expanded stretch of the channel), further illustrating the increasing of velocities and their influence over the drop in this region.

A channel with a forward-facing step is also studied in this section, as described in Fig. 3(b). In this case, the corner of the step is located at position $(0.35 ; 0.00)$. The same properties/features of the previous model is considered for the fluid subdomains and the BEM; however, the solid subdomain (i.e., the walls of the channel) is now defined by $E=10^{6}$ and $v=0.25$ (thus, a more stiff material is considered for the flexible channel), discretized by 840 square FE.

As before, and as depicted in Fig. 3(b), two drops are considered within the stepped channel, and their evolution through the channel considering different slip conditions is depicted in Figs. 10 and 11, taking into account rigid and flexible walls, respectively. The evolution of the horizontal position of the mass centre of the drops is depicted in Fig. 12, analogously to Fig. 7.

It can be observed that the introduction of the forward-facing step considerably reduces the velocity of the drops through the channel and, again, as expected, the deformations of the drops describe the velocity profile of the carrying fluid. It is interesting to observe that, for the horizontal channel, the expansion of its flexible walls implied into a reduction of the velocities of the drops whereas, for the stepped channel, an increase of these velocities is observed, once deformable walls are considered. This is probably because, once flexible walls are considered, the stepped channel configuration gets closer to the horizontal channel 
configuration, allowing an easier flow through its boundaries, and thus the velocities through the channel are increased.

As in all previous cases, greater velocities are obtained if nonlinear slip boundary conditions are considered, and considerably lower velocities are observed once the no-slip boundary condition is applied. This is, of course, expected since, for both channel geometries, non-zero velocity fields along the fluid-solid interface should allow a faster flow through the channel. However, faster flows are not always obtained once slip boundary conditions are considered, as illustrated in the next application.

\subsection{Lid-driven cavity flow}

In this section, a lid-driven cavity flow is considered. A sketch of the model is provided in Fig. 13. The geometry of the square cavity is defined by a length of 1 and a thickness of 0.02 (vertical and lower horizontal walls). The upper horizontal wall stands for a moving boundary, which is defined by a prescribed unity horizontal velocity. The physical properties of the model are: $\mu^{f}=10^{-3} ; \mu^{d}=5 \cdot 10^{-4} ; \gamma=10^{-3} ; E=10^{5} ; v=0.25$. The external contour of the bulk fluid is discretized by $200 \mathrm{BE}$ and each drop surface is discretized by $30 \mathrm{BE}$; the vertical and lower horizontal walls of the cavity are discretized by 608 square FE. Each drop has a diameter of 0.2 and their centres are located at $(0.00 ; 0.00)$ for the first analysis, and at $(0.20$; $0.35),(0.20 ; 0.00)$, and $(0.20 ;-0.35)$, for the second analysis. A time-step of $2 \cdot 10^{-2}$ is adopted for all the analyses.

As in the first application, initially, rigid walls and no drops are considered within the bulk fluid, allowing obtaining the velocity profiles along the boundaries of the model. The results obtained are depicted in Fig. 14. For the case of no-slip boundaries, a unity horizontal velocity is applied at the top horizontal boundary, whereas zero velocities are applied at the other boundaries (prescribed boundary conditions). As expected, this configuration is modified once slip boundary conditions are considered. In this case, reduced velocities are obtained along the top boundary (with a maximum value of around 0.6) and non-zero values arise on the other boundaries. Thus, the introduction of slip boundary conditions on the boundaries of the cavity homogenises the flow within it, smoothing the fluxes along the model. As a consequence, velocities are reduced next to the top of the model, and increased next to the bottom and vertical borders. In addition, it is interesting to observe that, for this cavity model, basically the same results are obtained considering linear and nonlinear slip boundary conditions; thus, only the linear model is further employed in the analyses that follow. 
In order to study the evolution of drops inside the cavity, initially, a single drop is considered, initially positioned at the centre of the cavity. The evolution of the deformation of the drop is depicted in Fig. 15, considering slip and no-slip boundary conditions, as well as rigid and flexible walls. As expected, the movement of the drop follows the velocity profile of the bulk fluid, and the figure indirectly illustrates these profiles. As previously observed, taking into account no-slip boundary conditions, greater velocities are obtained next to the top of the model, and thus the drop moves faster in Figs. 15(a) and (c). In addition, a less smooth movement of the drop is observed considering the no-slip condition and trajectories with shaper angles are obtained, as well as more intense deformations of the geometry of the drop. Fig. 15 shows basically the same results for the deformation of the drop considering rigid and flexible walls. In fact, taking into account slip boundary conditions, even the obtained deformation of the flexible walls is practically negligible, as depicted in Fig. 15(d). Thus, in the analyses that follow, only rigid walls are considered.

Once the movement of a single drop has been considered, more complex analyses can be carried out, and the interaction of several drops within the cavity can be studied. For this second group of analyses, a third configuration is also considered for the boundary conditions of the model in which slip boundary conditions are only considered on the bottom and vertical contours of the cavity whereas, in its upper boundary, a unity horizontal velocity is directly prescribed. Taking into account this configuration, the cavity is supposed not to be closed by a moving lid, but opened and linked to a flow that has unity horizontal velocity at that common boundary. In order to properly refer to each configuration that is focused here, the following nomenclature is adopted: (i) no-slip model - all boundaries of the cavity consider no-slip boundary conditions; (ii) slip model - all boundaries consider slip boundary conditions; and (iii) mixed model - the lower horizontal and the vertical boundaries consider slip boundary conditions and the upper horizontal boundary considers no-slip boundary conditions.

Computed results are depicted in Fig. 16, taking into account three drops (initially located as described in Fig. 13), and the above-discussed three configurations of boundary conditions. The evolution of the deformation of each drop is separately depicted in Fig. 17, illustrating the computed results in more detail. As observed in these figures, very distinct results are obtained, according to the configuration in focus. For the no-slip model, the drop initially located at $(0.20$; 0.35$)$ moves very fast, and its trajectory is greatly influenced by the drop initially located at $(0.20 ; 0.00)$. This second drop has a trajectory with very sharp angles, and 
it is greatly deformed throughout the analysis. The third drop (initially located at position (0.20; -0.35)) moves very slowly, and it has a minor influence over the movement of the other two drops. For the slip model, on the other hand, the deformations of the drops are smoother. In this case the first two drops still deeply interact with each other, but their deformations and velocities are lower than in the previous case, and their trajectories are also less sharply bent. As expected, the reduction of the velocity of the third drop is not observed (as it is the case for the first and second drops), since this drop is located very close to the bottom of the model, where non-zero boundary velocities arise. For the mixed model, an intermediary behaviour occurs, considering the first two drops and the previously discussed models. In this case, these two drops move fast, similarly to what occurs when considering the no-slip model, but smoother deformations are then developed, in resemblance to what occurs considering the slip model. In contrast, a huge difference in the results takes place considering the third drop. For the mixed model, the third drop moves considerably faster than in the other models and its more intensive influence over the behaviour of the other two drops occurs sooner. Considering these basic aspects, the results depicted in Figs. 16-17 are in agreement to what is expected, taking into account these complex models. In fact, as one can observe, the proposed techniques allow intricate configurations to be considered, enabling complex analyses to be carried out.

\section{Conclusions}

In this work, fluid-fluid-solid interaction models are considered, taking into account boundary and finite element discretizations. The fluid subdomains are modelled by the BEM and the solid subdomains are modelled by the FEM. For the fluid-fluid coupling, a BEM subregion approach is considered, and remeshing is adopted along the common interfaces. For the fluidsolid coupling, a pseudo-coupled formulation is adopted, allowing each subdomain of the model to be solved separately, taking into account an uncoupled formulation through the time stepping procedure. Thus, smaller systems of equations are obtained and proper solvers are allowed to be directly employed within each subdomain of the model, according to the specific features of the BEM and FEM systems of equations, enabling more efficient analyses. Discontinuity of tractions are allowed on the fluid-fluid common interfaces through non-zero surface tension coefficients as well as discontinuity of velocities are allowed on the fluid-solid common interfaces through slip boundary conditions. Here, linear and nonlinear slip boundary conditions were considered, and a relaxed iterative formulation is proposed in order 
to deal with the nonlinear model, ensuring the convergence of the iterative analysis. Quasisteady/static formulations are focused, and the adopted time marching procedures are based on simple finite difference expressions.

Numerical results are presented in section 3, demonstrating the performance of the proposed methodologies for the analyses of complex configurations. Interacting drops are then studied, flowing through a carrying fluid bounded by rigid or flexible walls. Different geometrical and physical properties are considered, as well as different boundary conditions. The obtained results are in accordance to what is physically expected and in very good agreement to analytical solutions for simpler models where these exact solutions are available. Substantially different results may be obtained according to the parameters/configurations that are applied into the analysis, highlighting the importance of fully developed numerical approaches to properly handle the simulation of complex multiphysics phenomena, and the methodologies proposed here stand as a step forward in this direction.

\section{Acknowledgements}

The financial support from CNPq (Conselho Nacional de Desenvolvimento Científico $e$ Tecnológico), FAPEMIG (Fundação de Amparo à Pesquisa do Estado de Minas Gerais) and CAPES (Coordenação de Aperfeiçoamento de Pessoal de Nível Superior), Brazil, is gratefully acknowledged.

\section{References}

[1] H. A. Schwarz, Ueber einige Abbildungsaufgaben, Journal für fie Reine und Angewandte Mathematik, 70: 105-120, 1869.

[2] M. J. Gander, Schwarz methods over the course of time, Electronic Transactions on Numerical Analysis, 31: 228-255, 2008.

[3] L. Ling and E. J. Kansa, Preconditioning for radial basis functions with domain decomposition methods, Mathematical and Computer Modelling, 40: 1413-1427, 2004.

[4] V. Dolean, M. El Bouajaji, M. J. Gander, S. Lanteri, and R. Perrussel, Domain decomposition methods for electromagnetic wave propagation problems in heterogeneous media and complex domains, in Domain Decomposition Methods in Science and Engineering, 15-26, Springer, Berlin, 2011.

[5] J. R. Rice, P. Tsompanopoulou, and E. Vavalis, Interface relaxation methods for elliptic differential equations, Applied Numerical Mathematics, 32: 219-245, 2000.

[6] C.-C. Lin, E. C. Lawton, J. A. Caliendo, and L. R. Anderson, An iterative finite elementboundary element algorithm, Computers \& Structures, 59: 899-909, 1996. 
[7] Q. Deng, An analysis for a nonoverlapping domain decomposition iterative procedure, SIAM Journal on Scientific Computing, 18: 1517-1525, 1997.

[8] D. Yang, A parallel iterative nonoverlapping domain decomposition procedure for elliptic problems, IMA Journal of Numerical Analysis, 16: 75-91, 1996.

[9] W. M. Elleithy, H. J. Al-Gahtani, and M. El-Gebeily, Iterative coupling of BE and FE methods in elastostatics, Engineering Analysis with Boundary Elements, 25: 685-695, 2001.

[10] W. M. Elleithy and M. Tanaka, Interface relaxation algorithms for BEM-BEM coupling and FEM-BEM coupling, Computer Methods in Applied Mechanics and Engineering, 192: 29772992, 2003.

[11] B. Yan, J. Du, N. Hu, and H. Sekine, Domain decomposition algorithm with finite elementboundary element coupling, Applied Mathematics and Mechanics, 27: 519-525, 2006.

[12] Y. Boubendir, A. Bendali, and M. B. Fares, Coupling of a non-overlapping domain decomposition method for a nodal finite element method with a boundary element method, International Journal for Numerical Methods in Engineering, 73: 1624-1650, 2008.

[13] W. M. Elleithy, M. Tanaka, and A. Guzik, Interface relaxation FEM-BEM coupling method for elasto-plastic analysis, Engineering Analysis with Boundary Elements, 28: 849- 857, 2004.

[14] H. Z. Jahromi, B. A. Izzuddin, and L. Zdravkovic, A domain decomposition approach for coupled modelling of nonlinear soil-structure interaction, Computer Methods in Applied Mechanics and Engineering, 198: 2738-2749, 2009.

[15] D. Soares Jr. and L. Godinho, Inelastic 2D analysis by adaptive iterative BEM-FEM coupling procedures, Computers and Structures,156: 134-148, 2015.

[16] D. Soares Jr., Fluid-structure interaction analysis by optimized boundary element - finite element coupling procedures, Journal of Sound and Vibration, 322: 184-195, 2009.

[17] D. Soares Jr., Numerical modeling of acoustic-elastodynamic coupled problems by stabilized boundary element techniques, Computational Mechanics, 42: 787-802, 2008.

[18] D. Soares Jr., Coupled numerical methods to analyze interacting acoustic-dynamic models by multidomain decomposition techniques, Mathematical Problems in Engineering, Article ID 245170, 28 pages, 2011.

[19] D. Soares Jr. and L. Godinho, An overview of recent advances in the iterative analysis of coupled models for wave propagation, Journal of Applied Mathematics, Article ID 426283, 21 pages, 2014.

[20] S. Gümgüm and L. C. Wrobel, DRBEM formulation for transient Stokes flow with slip boundary condition, Engineering Analysis with Boundary Elements, in press.

[21] S. Granick, Motions and relaxations of confined liquids. Science, 253: 1374-1379, 1991.

[22] C. Neto, V. Craig and D. R. M. Williams D.R.M., Evidence of shear-dependent boundary slip in Newtonian liquids, The European Physical Journal E: Soft Matter and Biological Physics, 12: 71-74, 2003.

[23] C. Neto, D. R. Evans, E. Bonaccurso, H. Butt and V. Craig, Boundary slip in Newtonian liquids: A review of experimental studies, Reports on Progress in Physics, 68: 2859-2897, 2005.

[24] M. T. Matthews and J. M. Hill J.M., Newtonian flow with nonlinear Navier boundary conditions, Acta Mechanica, 191: 195-217, 2007.

[25] P. A. Thompson and S. M. Troian, A general boundary condition for liquid flow at solid surfaces, Nature, 389: 360-362, 1997. 
[26] L. S. Kuo and P. H. Chen, A unified approach for nonslip and slip boundary conditions in the lattice Boltzmann method, Computers and Fluids, 38: 883-887, 2009.

[27] H. Power, J. Soavi, P. Kantachuvesiri and C. Nieto, The effect of Thompson and Troian's nonlinear slip condition on Couette flows between concentric rotating cylinders, Z Zeitschrift für angewandte Mathematik und Physik, 66: 2703-2718, 2015.

[28] L. C. Wrobel, D. Soares Jr and C. L. Das Bhaumik, Drop deformation in Stokes flow through converging channels, Engineering Analysis with Boundary Elements, 33: 993- 1000, 2009.

[29] C. A. Brebbia, J. C. F. Telles and L. C. Wrobel, Boundary Element Techniques. SpringerVerlag, Berlin, 1984.

[30] L. C. Wrobel and M. H. Aliabadi, The Boundary Element Method, vols. I and II, Wiley, Chichester, 2002.

[31] T. J. R. Hughes, The Finite Element Method - Linear Static and Dynamic Finite Element Analysis. Dover Publications, Mineola, New York, 2000.

[32] O. C. Zienkiewicz and R. L. Taylor, The Finite Element Method, vols. I and II, McGraw-Hill, London, 1989.

[33] M. T. Matthews and J. M. Hill, Newtonian flow with nonlinear Navier boundary condition, Acta Mechanica, 191: 195-217, 2007.

[34] J. L. Bull, Cardiovascular bubble dynamics, Critical Reviews in Biomedical Engineering, 33: 299-346, 2005.

[35] J. L. Bull, The application of microbubbles for targeted drug delivery, Expert Opinion on Drug Delivery, 4: 475-493, 2007.

[36] J. Calderon, B. Eshpuniyani, J. B. Fowlkes, and J. L. Bull, A boundary element model of the transport of a semi-infinite bubble through a microvessel bifurcation, Physics of Fluids, 22: 061902, 2010.

[37] B. Eshpuniyani, J. B. Fowlkes, and J. L. Bull, A boundary element model of microbubble sticking and sliding in the microcirculation, International Journal of Heat and Mass Transfer, 51: 5700-5711, 2008.

[38] C. Nieto, M. Giraldo and H. Power, Boundary integral equation approach for Stokes slip flow in rotating mixers, Discrete and Continuous Dynamical Systems, Series B, 15:1019-1044, 2011. 
(a)

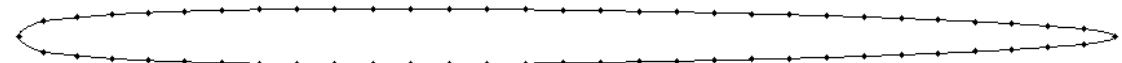

(b)

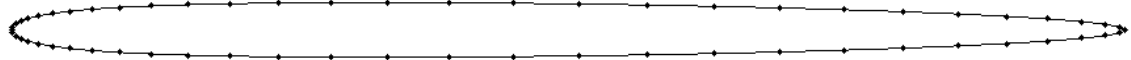

Fig.1 - BEM discretization of a deformed drop at a given time step:

(a) with remeshing; (b) without remeshing. 


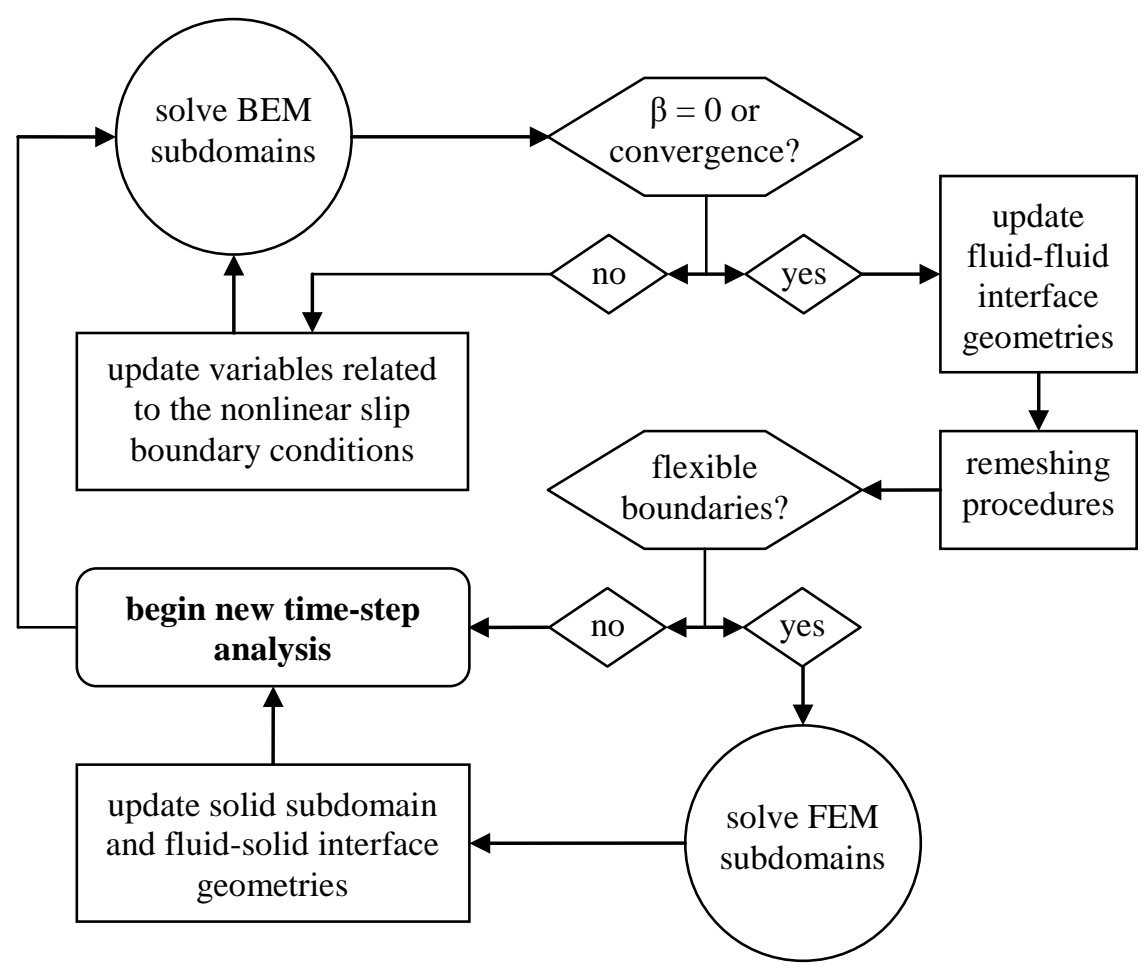

Fig.2 - Workflow diagram illustrating the sequence of the main steps of the proposed formulation. 

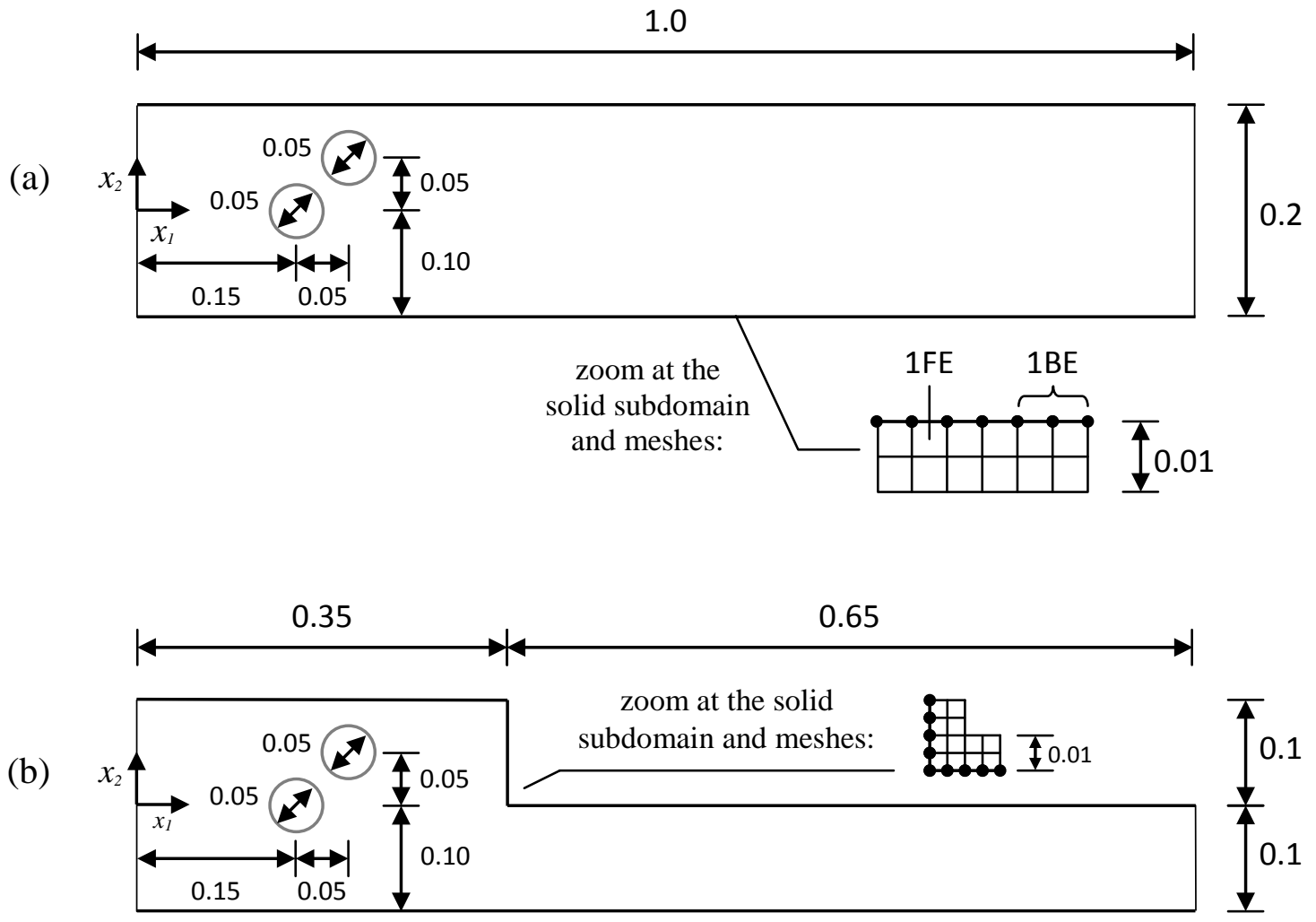

Fig.3 - Sketches of the channels and initial drops: (a) plain channel; (b) stepped channel. 


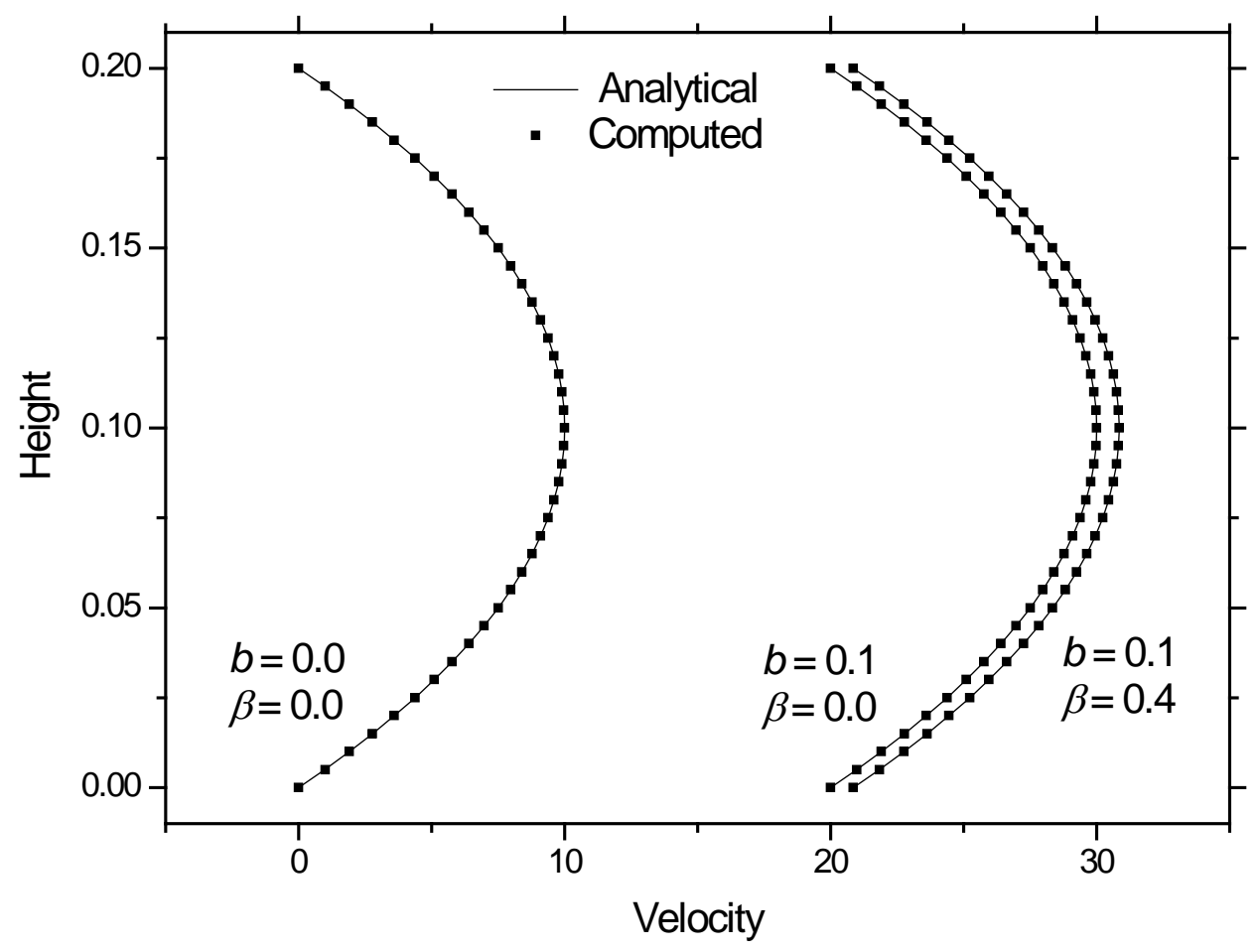

Fig.4 - Velocity profile for several slip boundary conditions (plain channel with rigid walls and no drops within). 
(a)

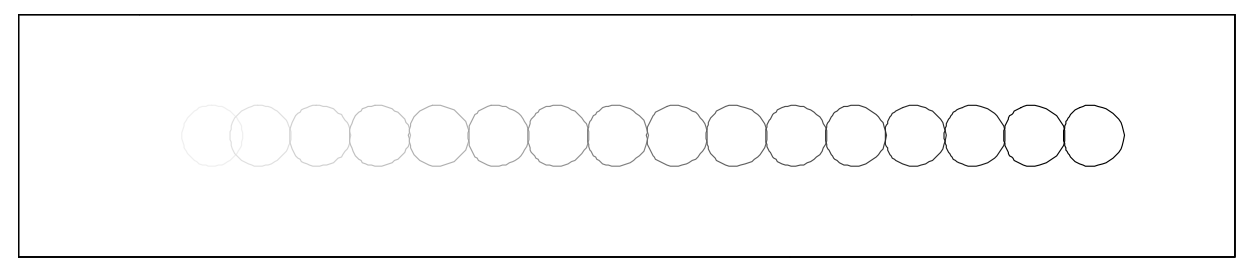

(b)

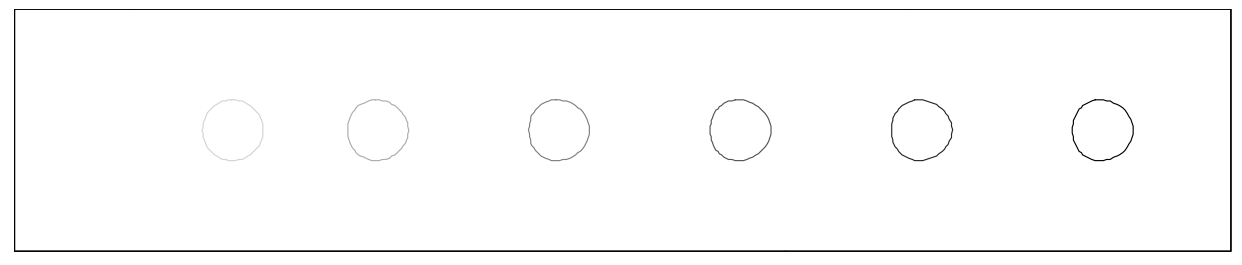

(c)

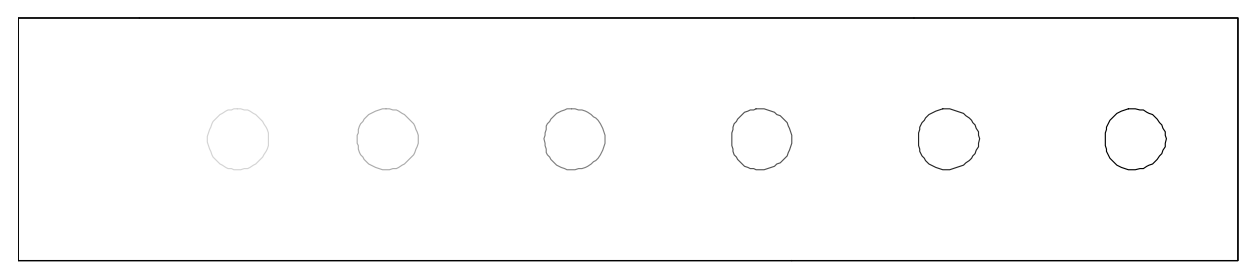

Fig.5 - Evolution of the deformation of one drop through a rigid plain channel considering different slip boundary conditions: (a) no-slip ( $b=0 ; \beta=0$ ); (b) linear $(b=0.1 ; \beta=0$ ); and (c) nonlinear $(b=0.1 ; \beta=0.4)$. Snapshots are depicted at each $5 \Delta t$. 
(a)

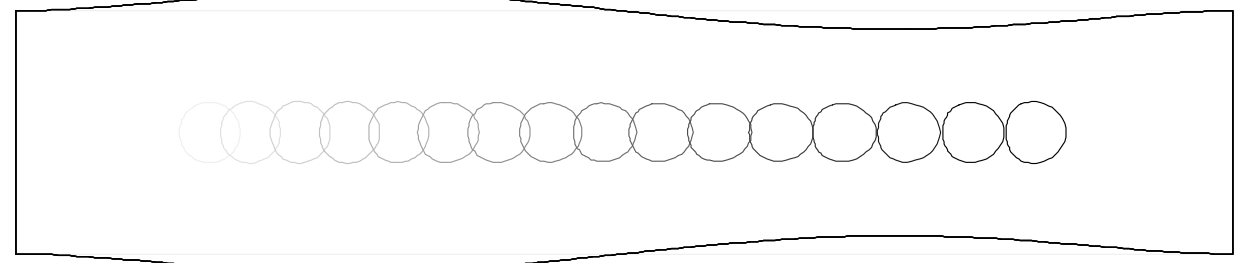

(b)

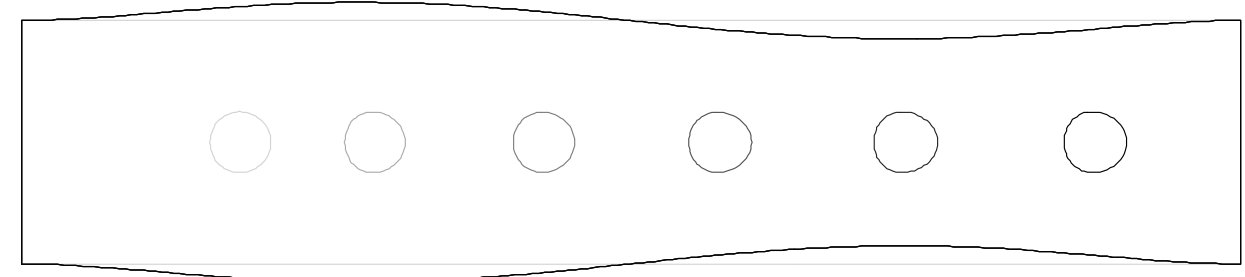

(c)

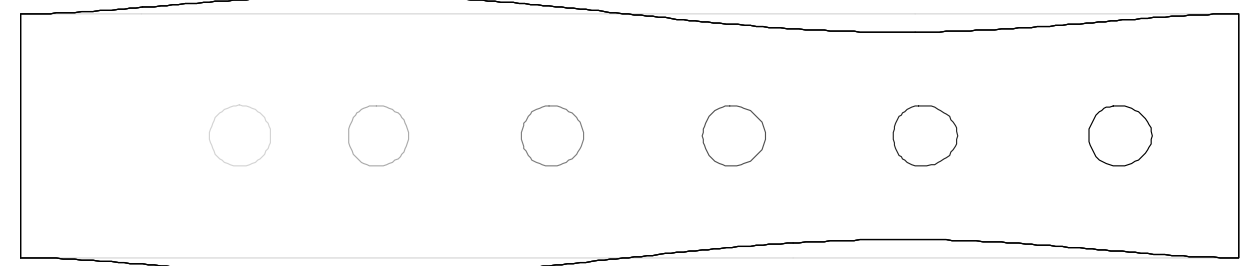

Fig.6 - Evolution of the deformation of one drop through a flexible plain channel considering different slip boundary conditions: (a) no-slip ( $b=0 ; \beta=0$ ); (b) linear ( $b=0.1 ; \beta=0$ ); and (c) nonlinear $(b=0.1 ; \beta=0.4)$. Snapshots are depicted at each $5 \Delta t$. 


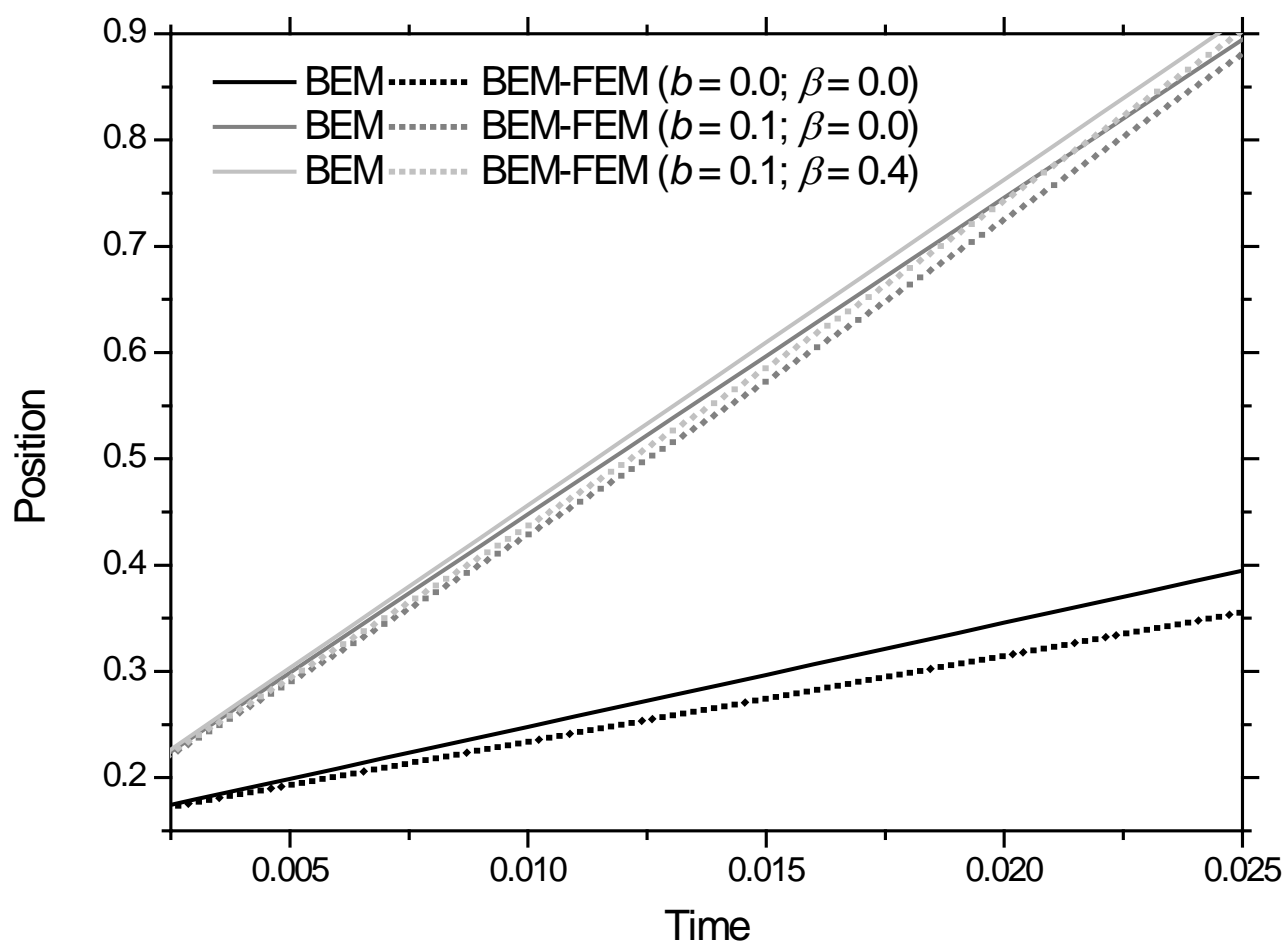

Fig.7 - Evolution of the horizontal position of the mass centre of the drop. 
(a)

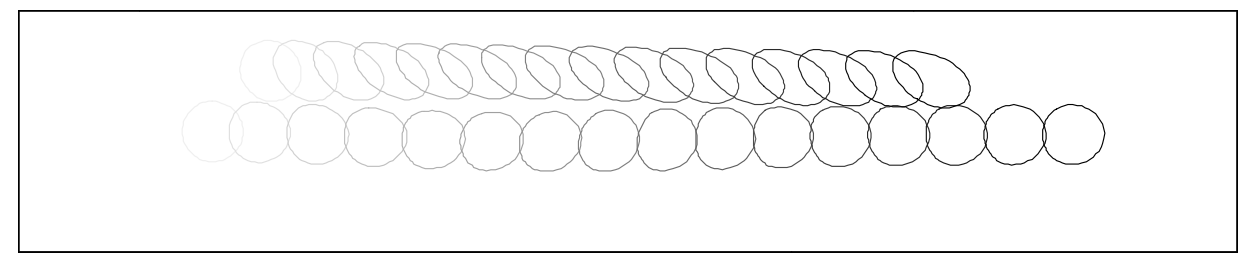

(b)

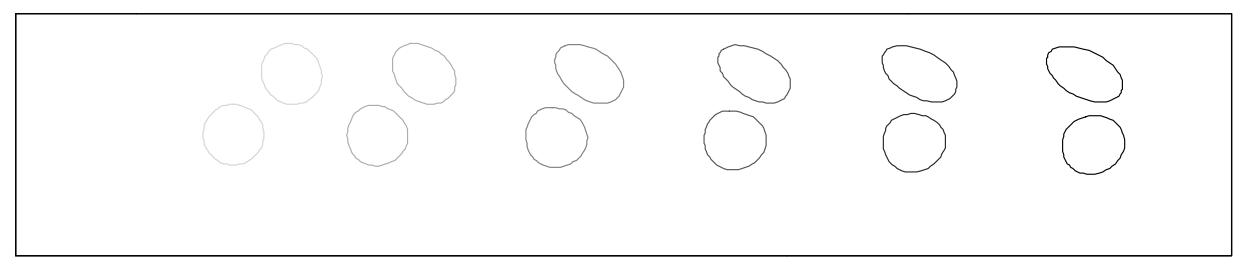

(c)

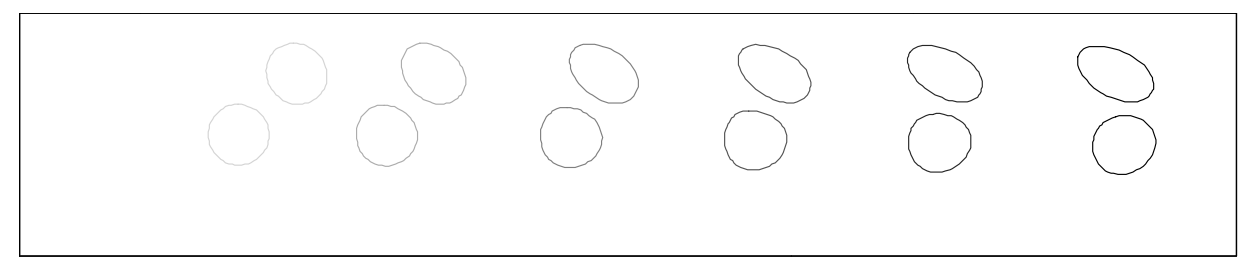

Fig.8 - Evolution of the deformation of two drops through a rigid plain channel considering different slip boundary conditions: (a) no-slip ( $b=0 ; \beta=0)$; (b) linear ( $b=0.1 ; \beta=0)$; and (c) nonlinear $(b=0.1 ; \beta=0.4)$. Snapshots are depicted at each $5 \Delta t$. 
(a)

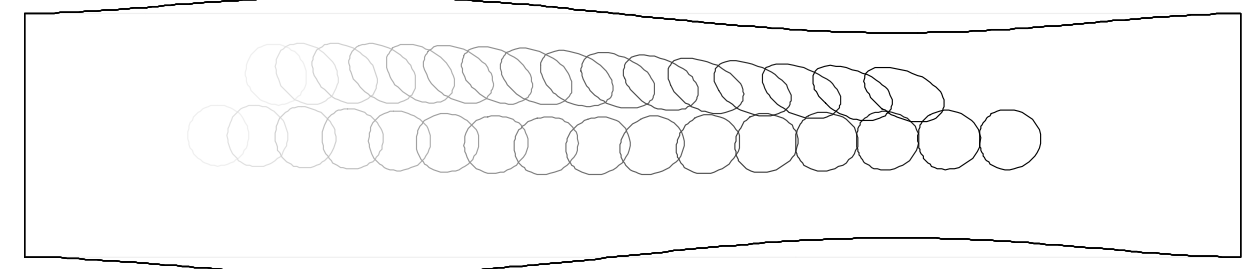

(b)

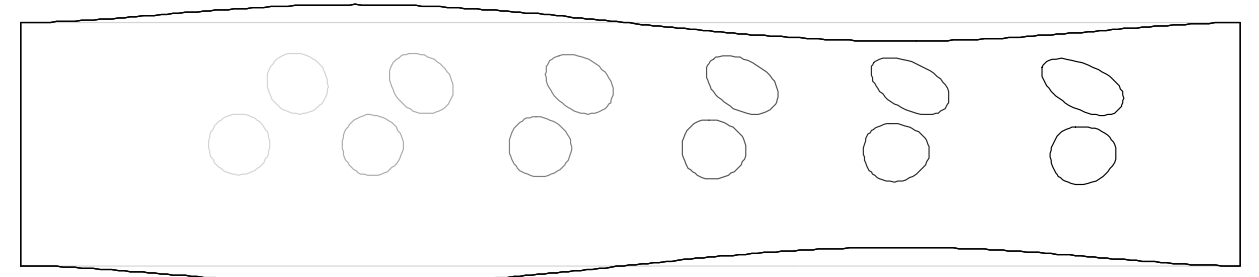

(c)

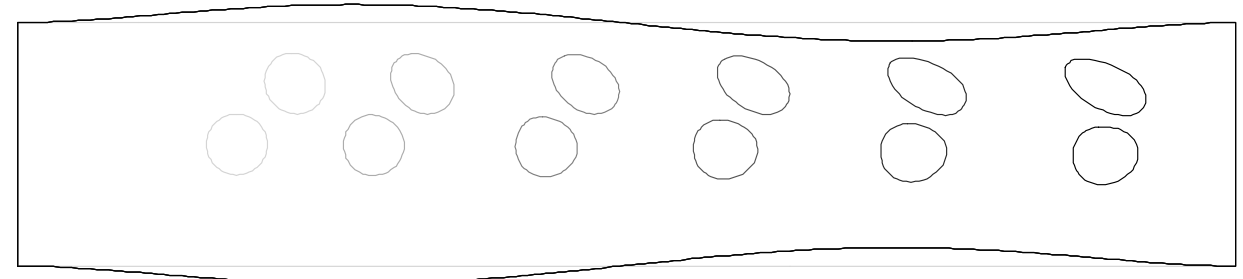

Fig.9 - Evolution of the deformation of two drops through a flexible plain channel considering different slip boundary conditions: (a) no-slip ( $b=0 ; \beta=0$ ); (b) linear ( $b=0.1 ; \beta$ $=0$ ); and (c) nonlinear ( $b=0.1 ; \beta=0.4)$. Snapshots are depicted at each $5 \Delta t$. 
(a)

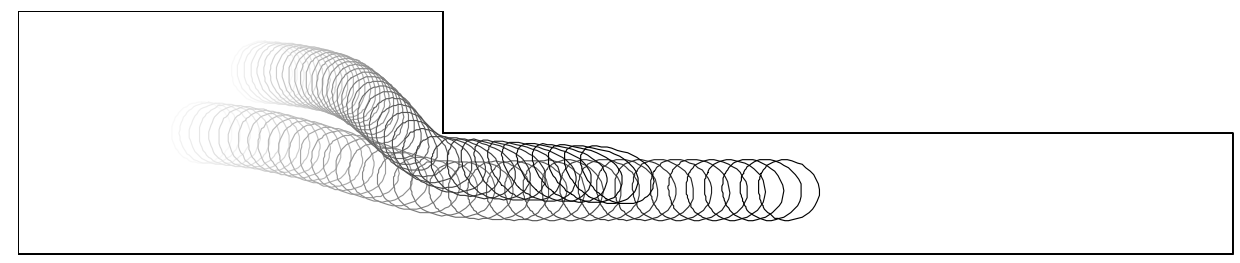

(b)

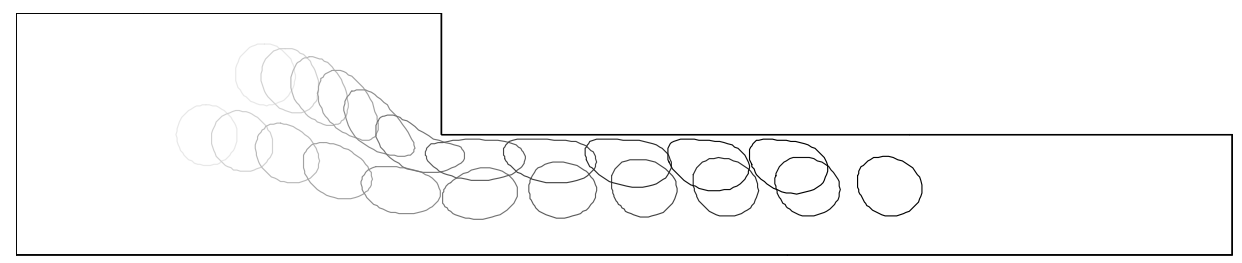

(c)

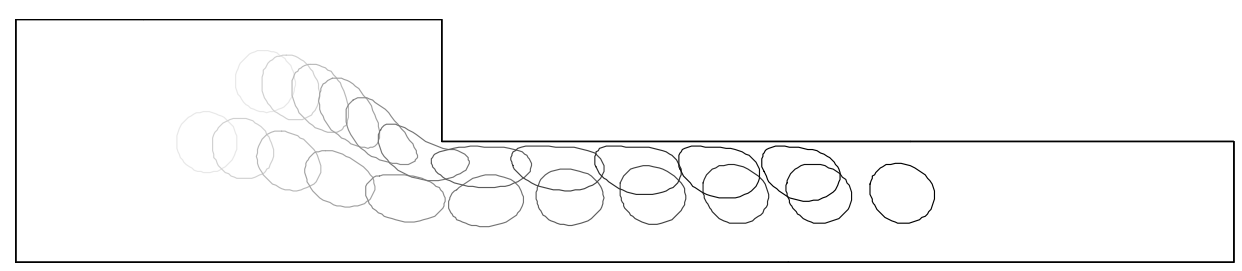

Fig.10 - Evolution of the deformation of two drops through a rigid stepped channel considering different slip boundary conditions: (a) no-slip ( $b=0 ; \beta=0$ ); (b) linear ( $b=0.1 ; \beta$ $=0)$; and (c) nonlinear $(b=0.1 ; \beta=0.4)$. Snapshots are depicted at each $5 \Delta t$. 
(a)

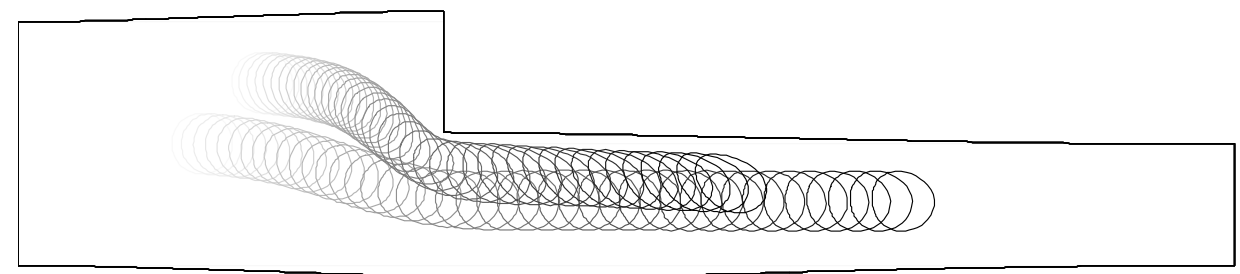

(b)

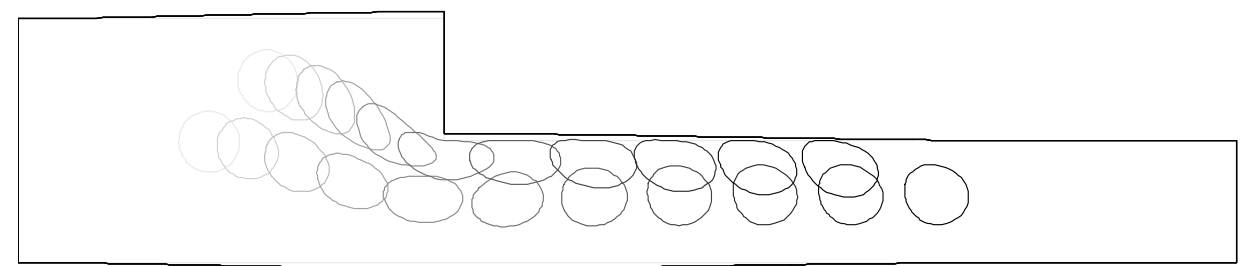

(c)

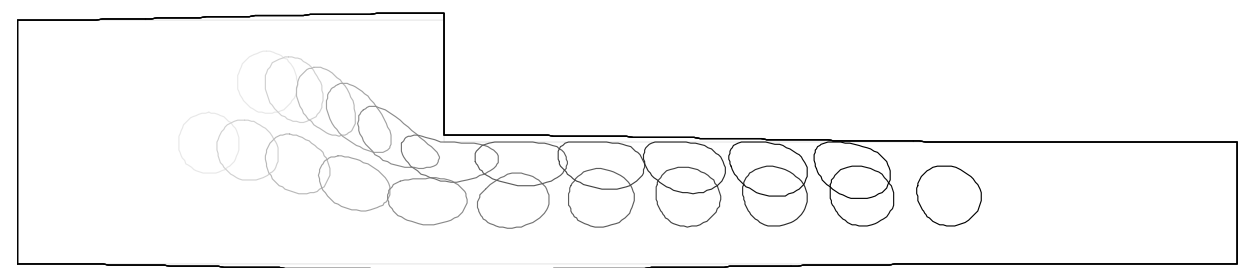

Fig.11 - Evolution of the deformation of two drops through a flexible stepped channel considering different slip boundary conditions: (a) no-slip ( $b=0 ; \beta=0$ ); (b) linear ( $b=0.1 ; \beta$ $=0)$; and (c) nonlinear $(b=0.1 ; \beta=0.4)$. Snapshots are depicted at each $5 \Delta t$. 
(a)

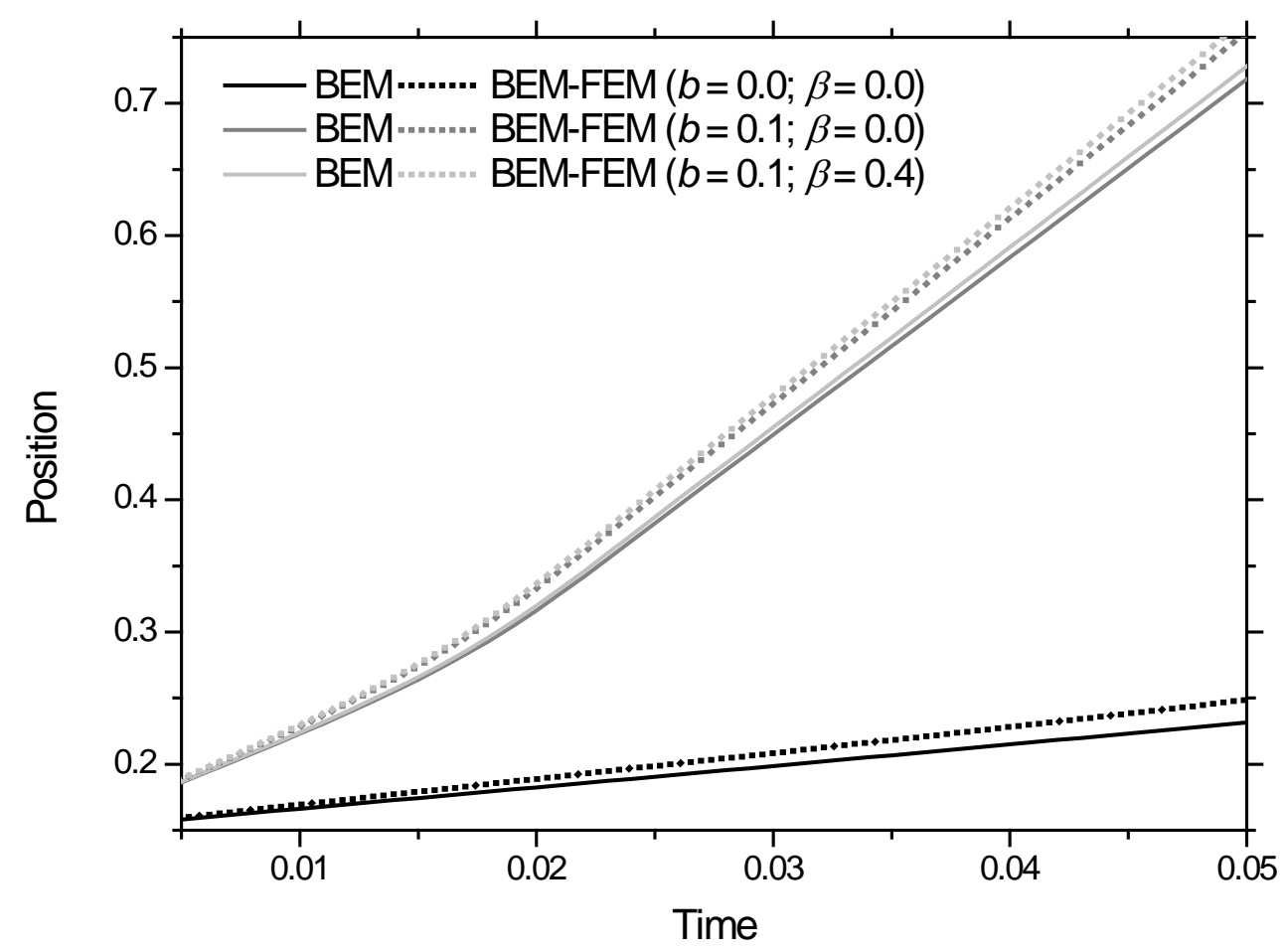

(b)

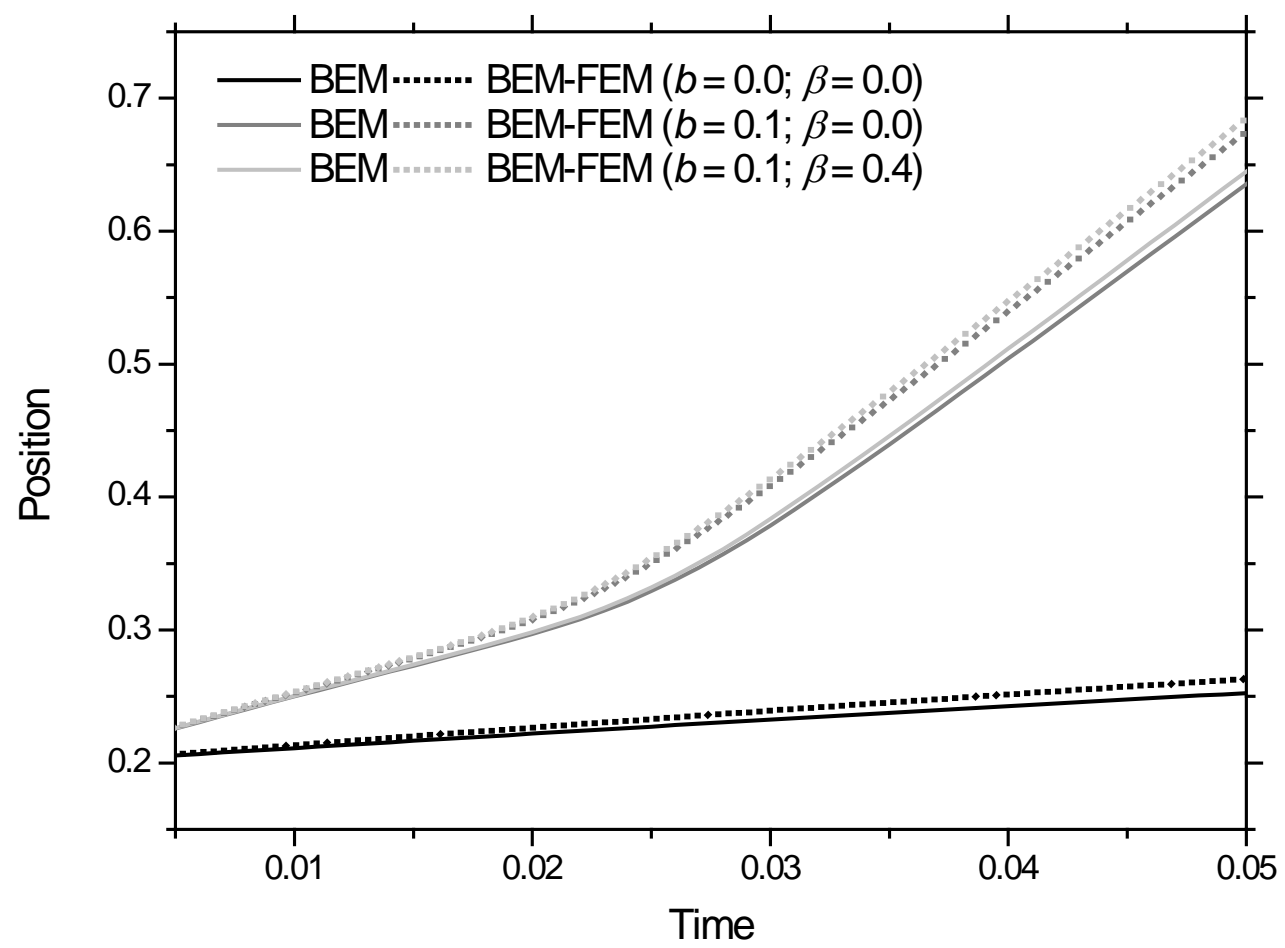

Fig.12 - Evolution of the horizontal position of the mass centre of the drops: (a) central drop; (b) upper drop. 


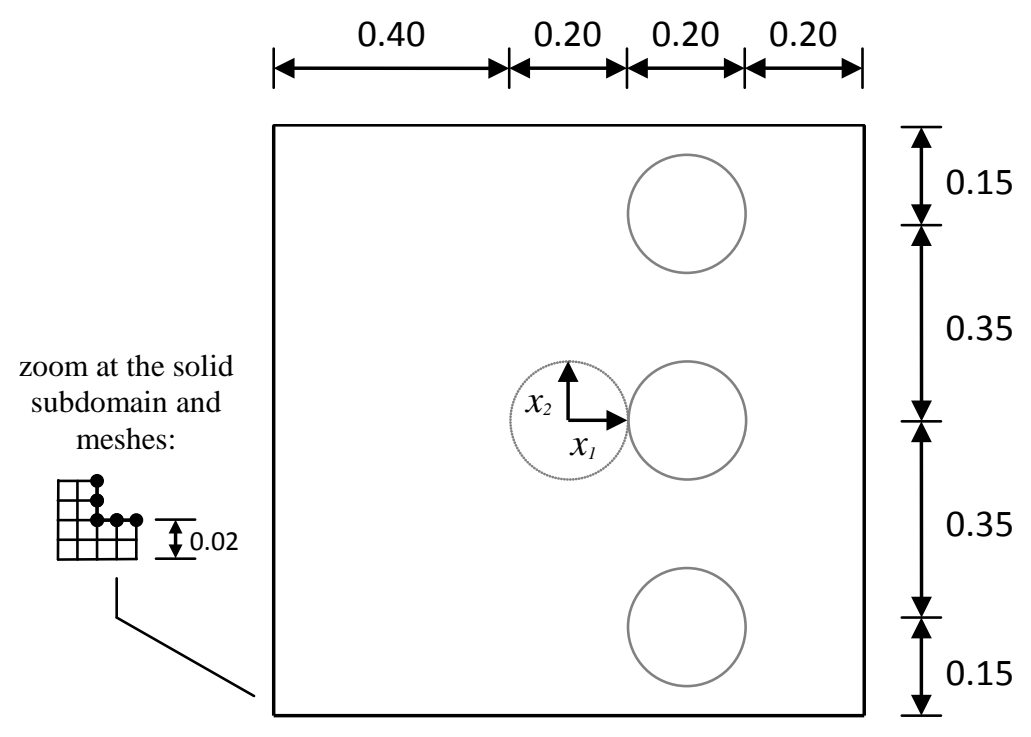

Fig.13 - Sketch of the cavity and initial drops: (i) first analysis - dot line drop; (ii) second analysis - solid line drops. 
(a)

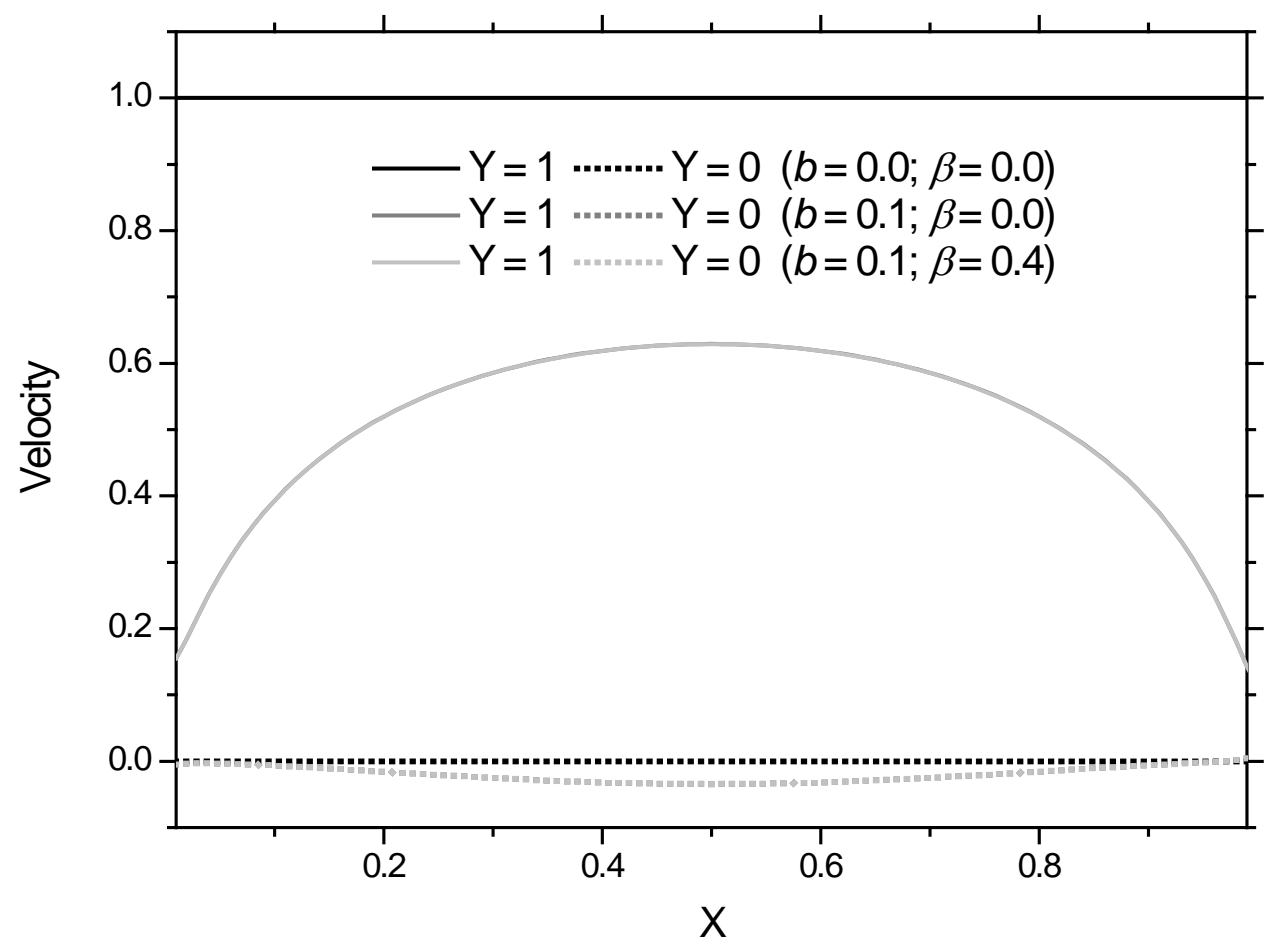

(b)

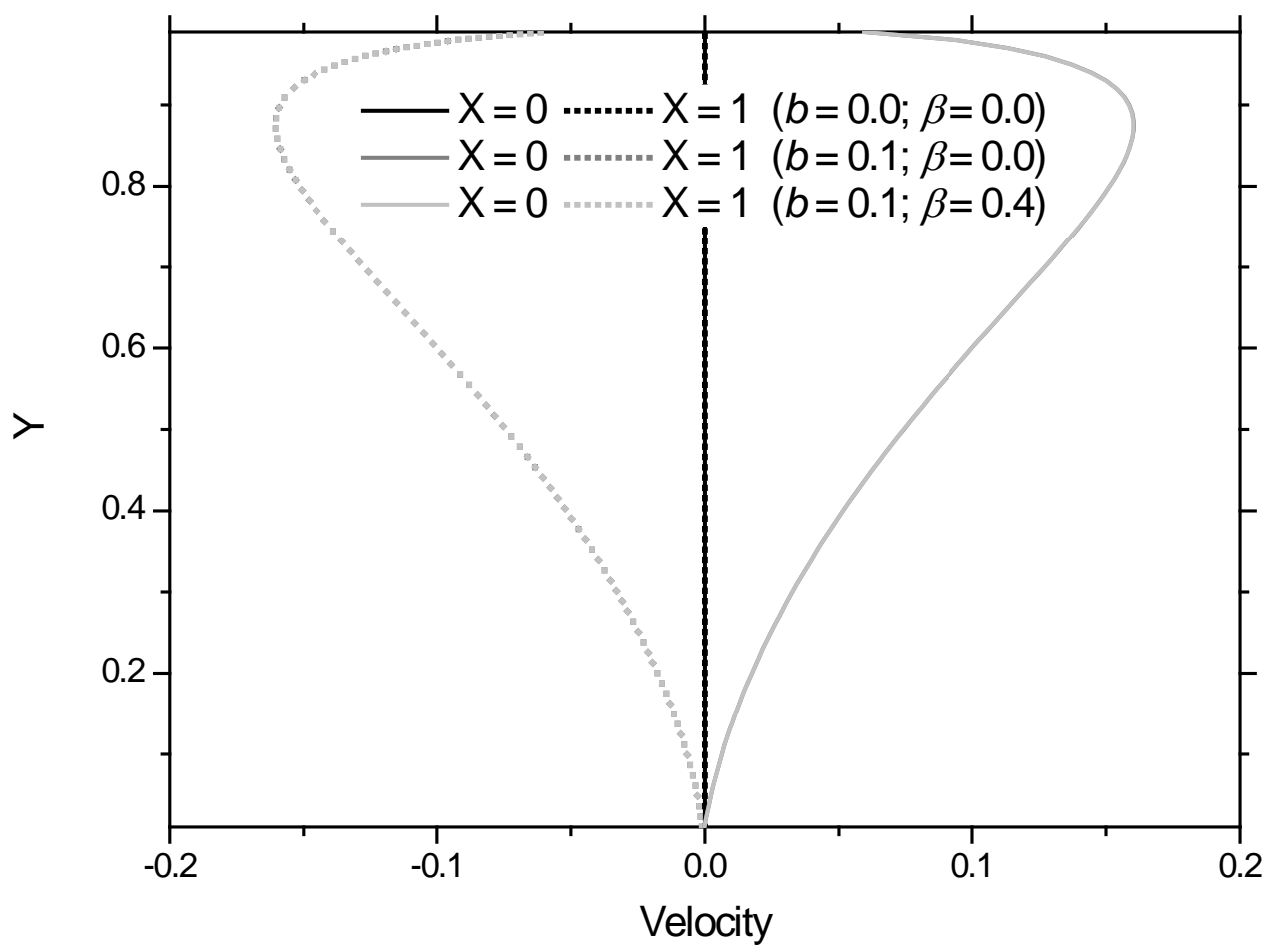

Fig.14 - Velocities along the boundaries of the model considering several slip boundary conditions (cavity with rigid walls and no drops): (a) horizontal walls; (b) vertical walls. 


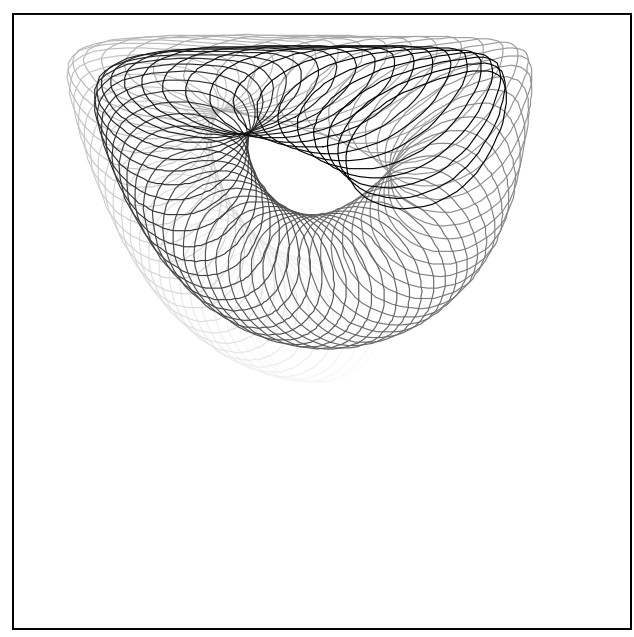

(a)

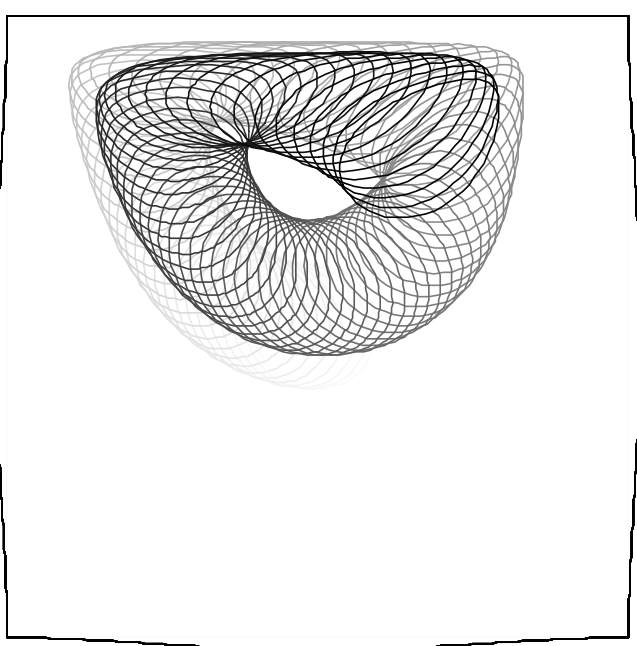

(c)

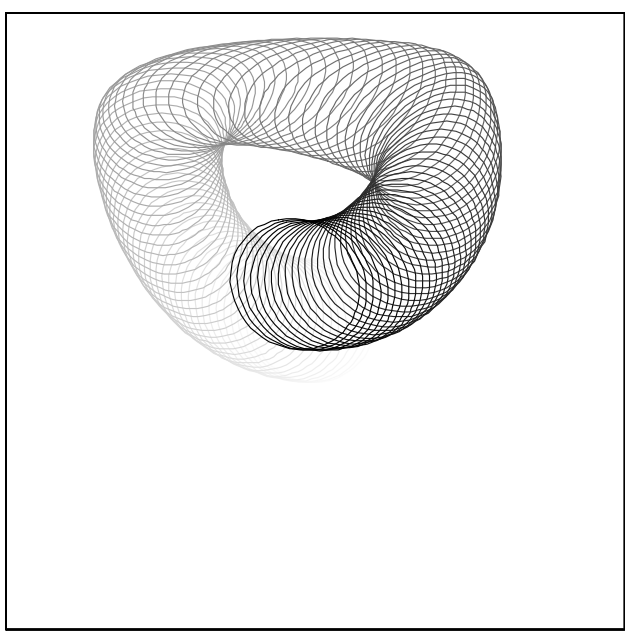

(b)

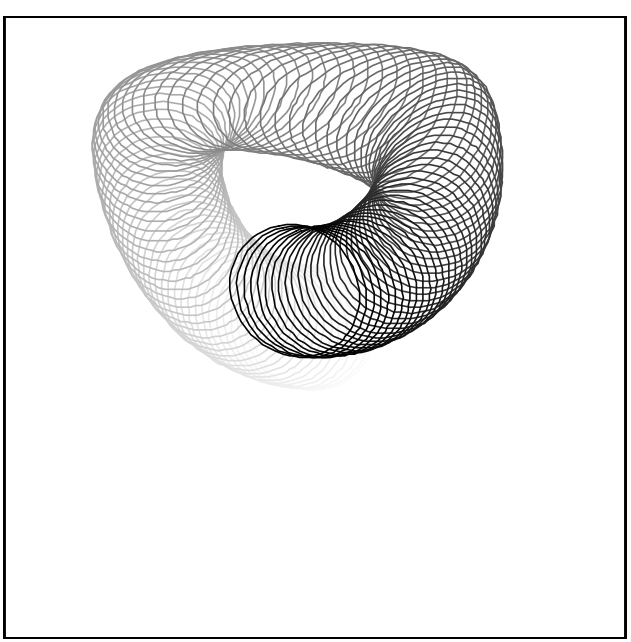

(d)

Fig.15 - Evolution of the deformation of one drop within the square cavity considering different slip boundary conditions and flexibilities for the walls: (a) no-slip ( $b=0 ; \beta=0$ ) and rigid walls; (b) slip ( $b=0.1 ; \beta=0)$ and rigid walls; (c) no-slip ( $b=0 ; \beta=0$ ) and flexible walls; and (d) slip ( $b=0.1 ; \beta=0)$ and flexible walls. Snapshots are depicted at each $5 \Delta t$. 
(a)

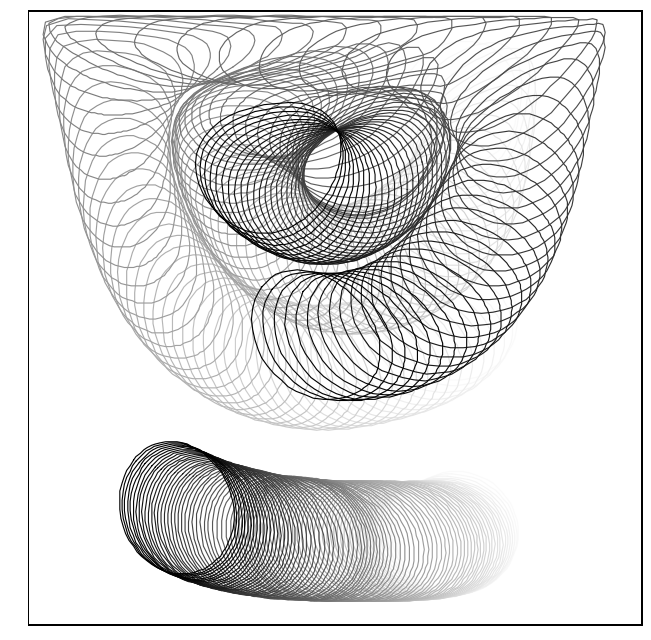

(b)

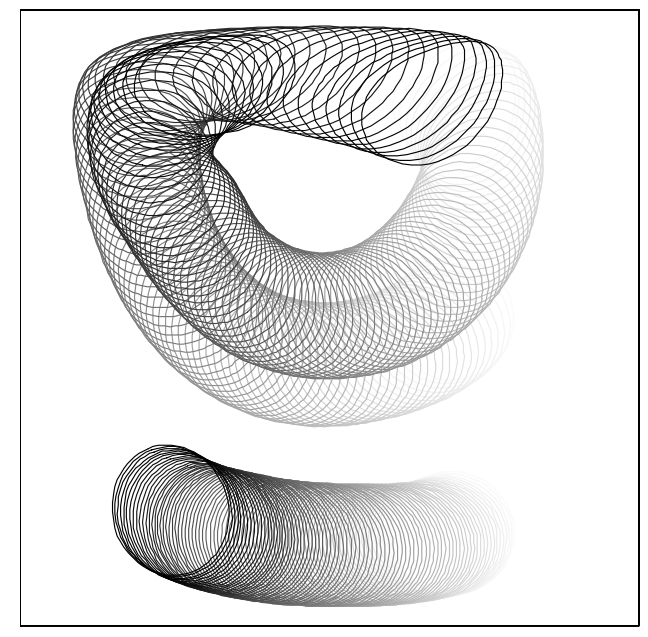

(c)

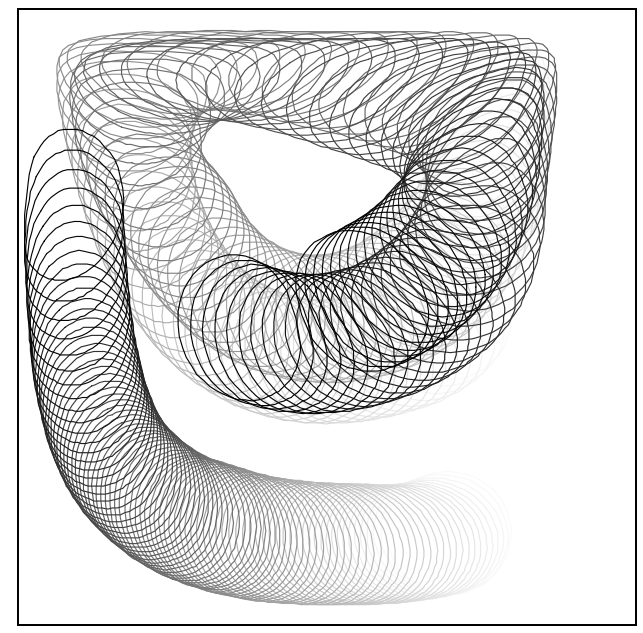

Fig.16 - Evolution of the deformation of three drops within the square cavity: (a) no-slip model; (b) slip model (c) mixed model. Snapshots are depicted at each $5 \Delta t$. 

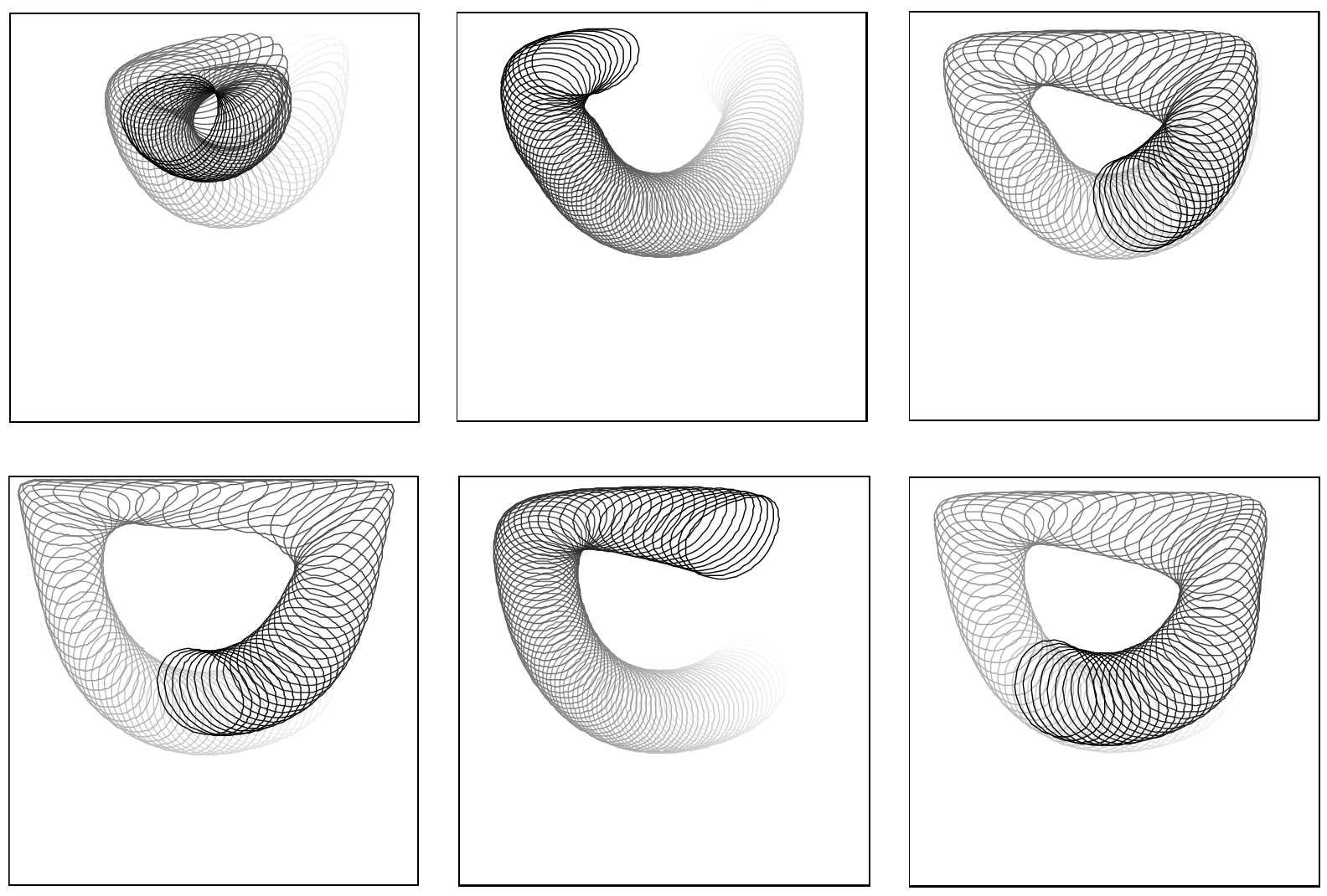

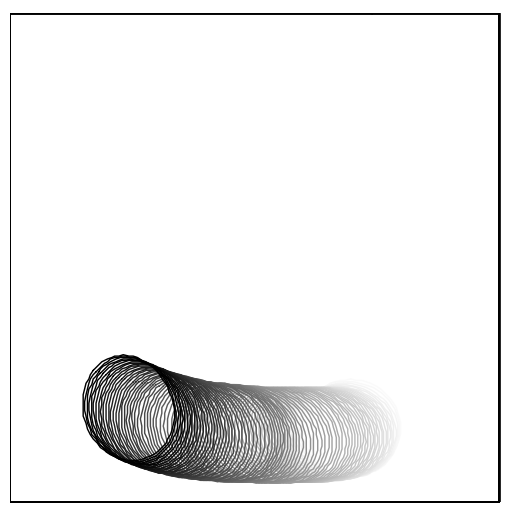

(a)

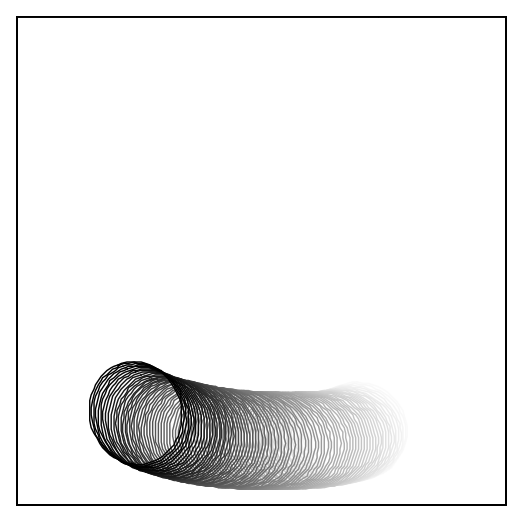

(b)

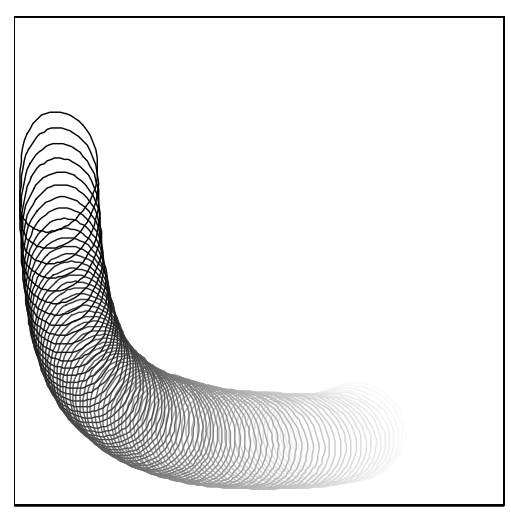

(c)

Fig.17 - Separated description of the deformation of each drop: (a) no-slip model; (b) slip model (c) mixed model. Snapshots are depicted at each $5 \Delta t$. 